\title{
UN ANÁLISIS BIBLIOMÉTRICO DE LA INVESTIGACIÓN \\ SOBRE MUJERES \\ IEN LA COMUNIDAD HISPANA
}

Mo Dolores Peris"

\section{EL ANÁLISIS BIBLIOMÉTRICO COMO MÉTRICA SOCIAL}

El Análisis bibliométrico tiene una gran conexión con el sociométrico, puesto que se trata de encontrar en la colectividad científica o social, las redes de influencia de las obras y acciones de un autor o grupo de autores, instituciones, revistas, escuelas o líneas temáticas. Parte del supuesto de que la ciencia es pública y está sujeta, como todo fenómeno social, a una organización que explica muchas de sus producciones, así como de sus omisiones. La bibliometría analiza las comunicaciones científicas, consideradas por algunos autores (Ziman, 1968) la dimensión social e institucional de una ciencia, en cuanto que tan sólo el reconocimiento de los colectivos de la comunidad, a través del conocimiento público, le aportan tal consideración.

El objeto material del análisis bibliométrico son las comunicaciones científicas, constituyendo la característica común que da nombre al amplio repertorio de índices métricos y técnicas de análisis que acumula. Desde la perspectiva social en que se orienta

\footnotetext{
* María Dolores Peris es Catedrática de Socioloía de la Universidad de Barcelona.
} 
este estudio, no es admisible esta total identificación de la ciencia con sus comunicaciones, diferenciándolas de la propia ciencia como sus producciones formales y públicas. De modo que el análisis bibliométrico puede considerarse como una vertiente del análisis de huellas, puesto que toda obra o publicación se interpreta desde la relación que sus características establecen con las circunstancias que la han originado y con las que a su vez potenciará. Los resultados bibliométricos representan así una medida de la función de eslabón que el objeto analizado ha tenido en la cadena de la evolución científica y social.

Un constructo nuclear del análisis bibliométrico, y que orienta sus aplicaciones en términos de investigación organizacional, ha sido el propuesto por Price (1961) bajo el nombre de Colegio invisible. Originariamente el término se refería a la red sociométrica de comunicaciones personales paralelas y previas a las publicaciones formales, así como a los liderazgos entre científicos. Ahora bien, cuando una nueva línea de investigación es publicada, las comunidades científicas que la siguen suelen establecer relaciones formales, además de las personales, y su detección y seguimiento a través de las redes de citaciones mutuas o por el análisis comparado de contenidos, permite detectar estructuras y líneas de influencia que no son accesibles desde otro tipo de estudios.La función de la bibliometría como amplificadora del conocimiento sobre los pequeños grupos científicos, muchos de ellos con proyectos creativos que apenas tienen influencia por falta de recursos o por la competencia de los equipos más poderosos, es en sí misma productiva, a tenor de la demostración, realizada ya en 1972 por Crane, de que la comunicación de conocimientos entre científicos acelera el desarrollo de una ciencia.

Pero además del análisis de redes de influencia científica, la bibliometría aporta muchos indicadores de aspectos de la sociedad científica y de su relación con las directrices políticas y coyunturales que explican la selección de muchos temas de interés en cada comunidad científica o grupos que rigen las instituciones científicas y docentes, así como los comités de publicaciones. Y permite también averiguar, mediante el análisis sistemático de las ausencias y no sólo de las presencias, las influencias por las que se impide la incorporación de determinados temas o recursos de investigación. Este estudio de las dependencias históricas, económicas, políticas o sociales de la ciencia es el objetivo de gran parte de las aplicaciones bibliométricas realizadas para averiguar las circunstancias que rodearon los orígenes o desarrollos de determinadas disciplinas o temas.

En este sentido guarda una proximidad metodológica y aplicada con los diversos tipos de análisis de contenidos, si bien el bibliométrico se orienta más hacia los aspectos 
materiales y formales de las publicaciones, que hacia los significados o desarrollos temáticos propiamente dichos. Por otra parte, el análisis de contenido suele limitarse a un texto o conjunto de textos muy concretos, mientras que la diversidad y amplitud, tanto espacial como temporal, es una de las características del análisis bibliométrico. En general, el análisis de contenidos busca concomitancias, coincidencias sobre los aspectos más representativos y frecuentes. En cambio el análisis bibliométrico, si bien algunos autores también lo enfocan hacia los elementos más frecuentes y de fácil resumen, consigue su máxima riqueza cuando profundiza en los detalles menos evidentes y por ello desapercibidos desde estudios menos rigurosos. Precisamente estas peculiaridades son las que suelen estar relacionadas con las innovaciones y cambios alternativos a las tendencias dominantes.

La bibliometría se inició en Sociología con el análisis de la productividad científica y de la eminencia y fue desarrollada por Lazarsfeld y Thielens (1958) como una métrica social a través de diversos indicadores obtenidos de los materiales bibliográficos. Posteriormente Merton y Zuckerman (1971) la consideraron el método empírico propio de la Sociología de las ciencias. No se trata, en vista de las fechas citadas, de una técnica nueva. Pero como el resto de procedimientos de investigación empírica de los contenidos sociológicos, y especialmente de métrica social, en nuestro país todavía permanece prácticamente inédita. Revisaremos por ello en este primer trabajo, que inicia una serie de análisis bibliométricos, sus conceptos fundamentales.

Los desarrollos básicos se publicaron durante la década de los 60 y las aplicaciones han ido en aumento especialmente a partir de finales de los 70 , gracias a la difusión de dos instrumentos imprescindibles para este análisis: la informatización de los paquetes de análisis multivariante, que permitieron una rápida relación de gran cantidad de información a través del tiempo. Y la creación de los catálogos de publicaciones científicas, como el Science Citation Index o el Social Science Citation Index, que facilitaron el acceso a gran parte de la información que estas técnicas analizan. La reciente informatización e incremento de catálogos bibliográficos, al permitir copias instantáneas de los sectores de . información seleccionados, eliminando la tediosa y costosa búsqueda manual, abre una nueva etapa muy prometedora para los análisis bibliométricos. Estos catálogos están disponibles al público en forma de CDROM en la mayoría de centros de investigación, y son accesibles por las redes informáticas, de modo que la bibliografía ha dejado de ser un indicador de dominio temático, y ha perdido su función de difusión de información, ya que con los actuales recursos todo usuario puede obtener en los pocos minutos que tarda en acceder a los menús, la más amplia y actualizada documentación sobre cualquier cam- 
po temático, por desconocido que le resulte. Actualmente las bibliografías pueden ser sustituidas muy ventajosamente por las direcciones electrónicas en las que se ha encontrado la información, pasando a ser más bien indicadores de rigidez e incapacidad adaptativa ante la innovación tecnológica. Características que por oponerse precisamente a las que definen la actividad científica, pueden también ser indicadores de la inadecuada funcionalidad de determinadas instituciones. Por todo ello, se puede pronosticar que pronto las largas bibliografías que tradicionalmente han seguido a los estudios científicos, serán sustituidas (deberían ser sustituidas) por introducciones bibliométricas que contextualicen adecuadamente el tema abordado, antes de iniciarlo.

El análisis bibliométrico se caracteriza, además de por el uso de fuentes bibliográficas como material de estudio, por sus dos enfoques metodológicos, según pretenda conocer la relación funcional con las circunstancias que en términos de precedentes o concomitantes rodean la obra o bien el seguimiento de su influencia. En consecuencia, se puede optar por diseños transaccionales o longitudinales, que a su vez determinarán el tipo de técnicas aplicadas, alternativas que explican la variedad de procedimientos utilizados. En estudios amplios es bastante común integrar las dos vertientes, pero en las publicaciones de artículos habitualmente se ha optado por un análisis bibliométrico parcial de tan sólo algunos de los indicadores.

\section{2. ÍNDICES DE PRODUCTIVIDAD}

En los primeros estudios, la evolución temporal fue el eje estructurador del análisis bibliométrico y se inició en torno a autores y al análisis de citas, puesto que la forma más fácil de seguir el impacto de una obra es a través de las referencias que recibe por parte de sus seguidores. Pero la productividad científica no sólo se puede analizar para los autores y equipos, sino también para los centros, proyectos y para las propias revistas, de forma que pronto se aplicó al análisis del impacto y difusión de las instituciones, integrándose bajo el nombre de Investigación de la productividad como parte esencial de los Métodos de evaluación de programas, ampliamente desarrollados en los años 70 . Y hablando de productividad científica, en cierta medida se puede afirmar que el análisis de la productividad bibliométrica fue el precedente más directo de los métodos de evaluación de programas y de medida del impacto. Los primeros diseños longitudinales del análisis de la evolución de la visibilidad y del impacto, tuvieron su alternativa en los que se limitaban a un corto periodo de tiempo, sustituyendo la evolución o comparación a través del tiempo, por la 
comparación entre diferentes entidades investigadas.Asimismo, junto a los indicadores cuantitativos de los que se obtienen los índices de productividad, aparecieron los cualitativos, de los que se obtienen otros índices como el de reputación.

Los índices de productividad han sido los más desarrollados, y entre los primeros obtenidos desde el análisis de citas está el propuesto por Platz en 1965 bajo el nombre de Índice de visibilidad, resultante del logaritmo de las citas que recibe el autor, autores $\mathrm{u}$ obra/s analizada/s. Su sustitución por el nombre de Índice de productividad es posterior (Gardfield E., Malin N.V. y H. Small, 1978) y consecuente al cambio de paradigma de las CCSS, resultando este nombre más coherente con el modelo input-output y más alejado de la detección de colegios invisibles como objetivo prioritario de la bibliometría.La distribución de publicaciones demostró que sigue la ley de Lotka, reajustada por Huber (1977) según la cual existe una relación inversamente proporcional entre el número de autores con $n$ trabajos y el cuadrado de dicho número $n$ de trabajos. Por ello la productividad se mide con una escala logarítmica, en una métrica equivalente a la de la discriminación perceptiva propuesta por Fechner. Esta progresión no aritmética aparece debido a que la productividad está mediatizada, como ya detectó Simon (1954), por diversos factores, entre los que destacan los recursos e integración en el mundo científico.En esta línea de la investigación de los factores relacionados con la productividad, cabe citar el estudio de Zuckerman (1977) que evidenció la bajada de productividad de los premios Nobel tras recibir el galardón, lo que hizo tomar en consideración tantolas variables situacionales como las motivacionales. Una línea igualmente cuantitativa intentó encontrar ponderaciones para las publicaciones cofirmadas, así como para la equivalencia entre trabajo de investigación y publicación, dada la redundancia con que muchos autores repiten la comunicación de los mismos resultados. Sin embargo no se han encontrado índices que sean totalmente aceptados y se suele seguir asociando trabajo con firma.

La crítica más frecuente a los indicadores obtenidos por la simple frecuencia cuantitativa de publicaciones sigue siendo que no tienen en cuenta la calidad de la producción. Una de las propuestas más consideradas en la diferenciación cualitativa ha sido la de Cole y Cole (1973), que clasificaron en tres categorías a los autores en función de la calidad de sus publicaciones: perfeccionistas (poco productivos pero valiosos), prolíficos (valiosos y productivos) y masivos o verborreicos (productivos sin calidad).Otra alternativa ha sido sustituir el índice de productividad por los de reputación (Cartter, 1966; Jones, Lindzey y Coggeshal, 1982; Roose y Andersen, 1970). No obstante, aunque el análisis bibliométrico utiliza prioritariamente estos índices, no se limita a ellos y recurre a una amplia gama de 
indicadores, que mediante los análisis multivariantes matizan las simples frecuencias, además de detectar las covariancias.Por ejemplo, la fecha de las citas aporta información de la actualización de conocimientos del autor. El número de firmantes, y desde un estudio más cualitativo su nombre, es una de las variables más frecuentemente incluídas. Las colaboraciones en las firmas de cada obra o la coparticipación en trabajos conjuntos es una importante fuente de análisis de redes y escuelas, considerando no sólo las frecuencias de colaboración, sino también los momentos, secuencias, equipos o aspectos como la dominancia de las especialiades y temas trabajados en común.

Cuando se aborda el análisis temático, hay que realizar algún tipo de clasificación, cuyo detalle dependerá de la envergadura del estudio. También puede incluirse un análisis de contenido de la obra, asociando la frecuencia de términos claves, o de los significados más habituales con las circunstancias en que se desarrollaron. Habitualmente en el análisis de autores suelen secuencializarse los temas según las épocas en las que se han desarrollado cada uno.

Otro indicador obligado son los canales de publicación, y en el caso de los libros se diferencia en cada obra tanto las primeras ediciones como las sucesivas reediciones y traducciones, con un seguimiento de las áreas lingüísticas a las que llega su impacto, al igual que las recensiones y comentarios recibidos.

En relación a los artículos cobra primordial importancia las revistas en que se incluyen. La productividad de una revista o centro editor se obtiene a través del número de citas que recibe en las publicaciones insertadas en otras revistas o libros. Las distribuciones de espacios, titulares y demás elementos formales se realiza como en los análisis de contenidos. A partir de los estudios acumulados sobre las revistas se han obtenido importantes conocimientos de los procesos sociales que median en la evolución de las ciencias. Incluso algunos especificados en términos de leyes bibliométricas, como la ley de Bradford. Esta ley predice la viabilidad de una especialidad científica a partir de la relación $1: n: n 2 \ldots$, donde $n$ es el número de revistas que ofrecen paulatinamente menor especialización sobre sus temas de estudio. Es decir, determina el número mínimo de fuentes fijas de información a las que todo lector debe tener acceso para poder estar actualizado, así como el número correspondiente de la red decreciente de revistas donde el tema de interés se va diversificando. La ley está validada por la evidencia de que un campo de especialización que no cuente con revistas propias a las que puedan acudir sus especialistas, tanto para leer como para publicar, de forma que los interesados necesiten diseminar ampliamente sus estudios, no tiene ningún porvenir. 
Pero además exige que el ámbito de especialización sea también considerado en otras revistas de mayor espectro, es decir, reconocido por sectores científicos con intereses parcialmente comunes o conectados. Lo que viene a determinar de nuevo la dependencia social de la ciencia, ya que tras las revistas científicas hay un grupo social, unas condiciones materiales y un área de influencia, además de una política científica, que explica el que sus contenidos no respondan exclusivamente a la lógica del conocimiento o del descubrimiento, ni siquiera a la historia ni a la oferta de trabajos por los investigadores, sino en gran medida a unas circunstancias sociales concretas. Circunstancias que de forma más o menos intencionada, determinan la evolución de unos conocimientos y la involución de otros.

\section{ANÁLISIS BIBLIOMÉTRICO DE LOS ESTUDIOS SOBRE MUJERES CONTENIDOS EN LA BASE DE DATOS DEL CSIC (1976 - 1997)}

\subsection{Información analizada}

Actualmente la Universidad de Barcelona cuenta en su red de Bibliotecas con tres bases bibliográficas que recopilan la práctica totalidad de publicaciones sociológicas de los últimos años: Social Sciences Citation Index, editada por el ISI con las publicaciones de 1400 revistas y editoriales desde el año 1986; Sociofile, que recopila las publicaciones de unas 1600 revistas de 55 países y 30 lenguas desde 1974. Y la que bajo el nombre del CSIC edita el Consejo Superior de Investigaciones Científicas, reuniendo un total de 6 bases de datos con aproximadamente 625.000 referencias de artículos publicados en más de 2.300 revistas editadas en España, así como los fondos de las diferentes bibliotecas del CSIC. Las 6 bases de datos son: CIRBICL (libros de las bibliotecas del CSIC); CIRBICR (revistas de las bibliotecas del CSIC); DATRI (Referencias de las transferencias de Resultados de Investigación y la oferta científica y tecnológica de la red OTRI/OTT); ICYT (artículos de investigación de la revistas de ciencia y tecnología editadas en España); IME (artículos publicados en las revistas médicas españolas) e ISOC (artículos de revistas de Ciencias Sociales y Humanidades editadas en España).

La red de Bibliotecas del CSIC que contienen fuentes de Humanidades y CCSS, y a las que se puede acceder electrónicamente con el código http://www.csic.es/cbic/huma.htm, con la variante /bibliote.htm para ampliar el campo a las demás bibliotecas de la red, son las Bibliotecas General y Central del CSIC, la Biblioteca de la Residencia de Estudiantes, el Instituto de Estudios Sociales Avanzados, el Instituto de Filosofía, el Instituto de Filolo- 
gía, el Instituto de Economía y Geografía, el Centro de Estudios Históricos y la Colección de Prehistoria en Madrid. El Instituto de Análisis Económico y el Instituto Milá y Fontanals en Barcelona. La Escuela de Estudios Hispanoamericanos en Sevilla, la Escuela de Estudios Árabes en Granada, el Instituto de Estudios Sociales Avanzados de Córdoba y el Instituto de Estudios Gallegos «P. Sarmiento» en La Coruña.Debido a esta amplia representatividad de las publicaciones de nuestro país y de los países hispanos se ha iniciado el estudio desde la tercera, con objeto de que una primera reducción y estructuración de la información permita abordar las otras dos con esquemas reductores de su amplitud elaborados desde nuestros propios referentes. Aunque estudios y publicaciones sobre la mujer se encuentran en otras bases de datos, especialmente en las clínicas, la base seleccionada es estrictamente sociológica y por tanto, los trabajos que aparecen de índole clínica o próxima a otras disciplinas, presentan un fuerte contenido relacionado con la sociología de la mujer.

La edición de la base consultada data de julio de 1997, si bien de este año no hay apenas incluídos estudios y los de 1996 son escasos, posiblemente porque los presupuestos de las citadas bibliotecas no habían permitido la actualización o porque los catálogos y la informatización no se realizan inmediatamente.En el análisis de esta primera base se ha procedido de forma totalmente exploratoria, sin esquemas previos que conformaran según prejuicios de autor el campo de la información analizada, obteniendo las dimensiones tan sólo a partir de las relaciones entre los contenidos. Este es el procedimiento más objetivo dado que tampoco se ha realizado ninguna selección, sino que se han analizado la totalidad de trabajos reunidos bajo el epígrafe MUJERES.

El total de artículos que al reclamar la clave MUJERES aparece en la base de datos es 1026. La posibilidad de copiarlos en un diskette para posteriormente someterlos a los diferentes procesos del análisis es decisivo para el tipo de estudios que aquí presentamos. La estructura de esta base es similar a las demás, contando con los campos que muestra la siguiente referencia:

* Documento

* Título:TRAYECTOS DOMICILIO-TRABAJO Y MODOS DE VIDA DE LAS MADRES ACTIVAS EL EJEMPLO DE LA AGLOMERACIÓN PARISINA

* Autores:FAGNANI, JEANNE

* Lugar-Trabajo:CNRS, PARIS, FRANCIA

Obra-Colectiva:AMERICA LATINA: PERFILES SOCIALES EN LOS NOVENTA

Revista:TREBALLS DE LA SOCIETAT CATALANA DE GEOGRAFIA 
* Datos-Fuente: 1988, 3 (13-14): 63-81, 16 REF

* Idioma:FR

* Tipo-Documento:AR

* Localización:ISOC

Clasificación:854030; 854020

Compilador-Director:PANIAGUA PEREZ, JESÚS ; VIFORCOS MARINAS, MARIA ISABEL

Congreso:JORNADAS LA MUJER LATINOAMERICANA EN LA MIGRACION INTERNACIONAL.1995. MADRID

Editor:LEÓN: UNIVERSIDAD DE LEÓN, 1993

ISBN:84-86842-86-7

Institución-Patrocinadora:FLACSO-SODEPAZ

Serie:SUPLEMENTOS CUADERNOS ÁFRICA-AMÉRICA LATINA. DOCUMENTOS DE TRABAJO

Notas:4 TABLAS, 1 MAPA, 5 GRÁFICOS

Señal:INT

* Descriptores:GEOGRAFÍA FEMINISTA ; EMPLEO ; MUJERES ; MERCADO DE TRABAJO ;CONDICIONES DE TRABAJO ; ESTRUCTURA PROFESIONAL ; LUGARES DE TRABAJO ;LUGARES DE RESIDENCIA ; MOVIMIENTOS PENDULARES

Identificadores:ASOCIACIÓN INTERNACIONAL EN CONTRA DEL RACISMO Y LA EXPLOTACIÓN SEXUAL:

Topónimos:PARÍS ; FRANCIA

Periodo-Histórico: 1900:1930;1940:1970;1970:1990

Siglo: 19

Resumen:....

Las líneas con asterisco son fijas. El resto se modifica según el tipo de estudio o publicación. Entre los campos variantes se encuentran: Otro-Idioma-Título; Obra-Colectiva; ISSN; Modo-Documento; Universidad; Legislación; Jurisprudencia y Décadas.De estos campos se han extraído, además de los contenidos temáticos que aportan tanto el título como los descriptores o palabras claves, y el nombre de la revista, la fecha de publicación, el número de referencías, de páginas así como el número de la revista y volumen, el idioma, lugar de trabajo del autor y topónimos, tres variables referidas a los autores: género, a partir del nombre, número de firmantes y al ordenarlos alfabéticamente, número de obras sobre la misma temática escritas por el mismo autor. La época se ha dicotomizado diferen- 
ciando los estudios actuales de los referidos a épocas anteriores a 1950, considerando que estudian generaciones pasadas. Los Centros de trabajo del autor e instituciones patrocinadoras se han combinado en la variable entidad. Al convertir en variables ficticias (dummy) los contenidos temáticos, se han totalizado 78 variables, que se analizan sectorialmente en función de la métrica utilizada.

Un análisis multidimensional de los descriptores que definen los principales contenidos de cada publicación, produjo el circumplex de la fig. 1 . Si bien el detalle de cómo se llegó a esta estructura cartesiana bipolar y su interpretación se ha tenido que aplazar a una segunda publicación, por evidentes limitaciones de espacio, se presenta por ser el resultado integrador del resto de índices obtenidos.

fig. 1. Circumplex que estructura en dimensiones bipolares los descriptores de las investigaciones sobre mujeres.

\section{Circumplex de descriptores de investigaciones sobre mujeres Modelo de distancia euclídea}

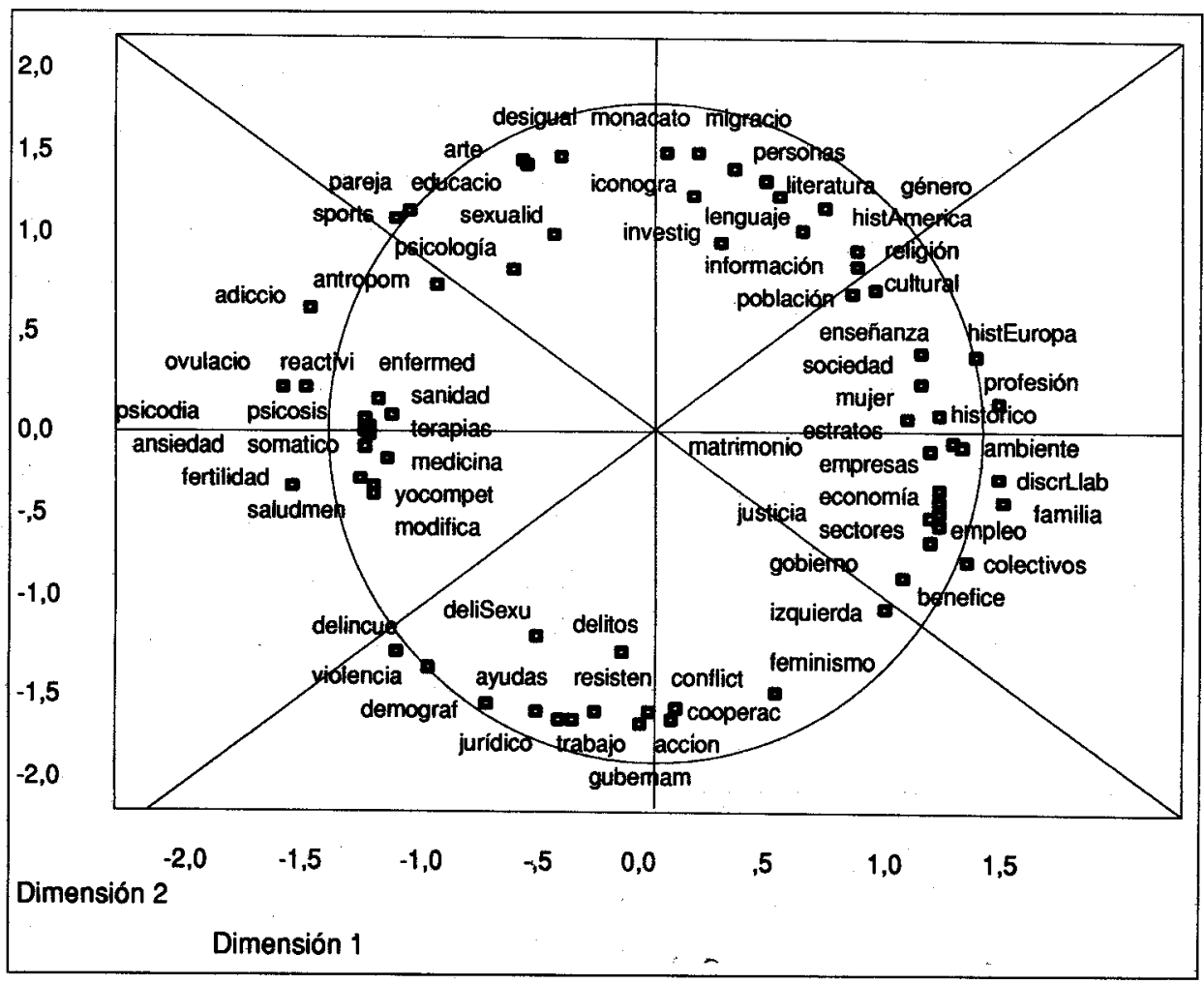




\section{REVISTAS EN LAS QUE SE PUBLICAN LOS ESTUDIOS SOBRE MUJERES}

Este estudio se ha realizado con ocasión del nacimiento de una nueva revista especializada en un área que está muy lejos de cumplir, en nuestro país, con los mínimos determinados por la ley de Bradford. Sea por ello bienvenida como instrumento necesario para la supervivencia de este sector de conocimientos, cuyas publicaciones en medios más próximos a los de difusión popular que a los científicos, llega a cuestionar incluso su carácter de ciencia..

El análisis bibliométrico aplicado pretende integrar los contenidos de la sociología de las mujeres, o si se desea, del género, en el más estricto método científico e incluso aplicaremos el principio de Fisher, partidario de proporcionar tan sólo los procedimientos y resultados de las investigaciones, sin ningún tipo de conclusiones, para que sea el lector quien libremente proceda a interpretarlos.

En cualquier caso, los estudios bibliográficos son abundantes en rèlaciones donde muestran el resultado de la selección y ordenamiento de la información, y especialmente redundantes cuando sustituyen la interpretación por la lectura de tablas.

Consideramos además que una ciencia debe diferenciar claramente el estado actual de conocimientos, de sus fundamentos y precedentes históricos. Por ello se ha seguido la evolución de esta temática, que a pesar de su brevedad muestra importantes cambios.

\subsection{Revistas incluídas en el estudio.}

En un total de 295 revistas diferentes se publican los 1026 estudios, lo que corresponde a uno 3,5 trabajos por revista. La ausencia de especialización temática en las publicaciones se evidencia en que tan sólo 16 publicaciones son las que aportan un número superior de 10 trabajos cada una, y la mayoría son publicaciones especializadas en otros campos científicos (psicología, medicina, geografía, ...). El número de revistas crece conforme se reduce el número de publicaciones sobre la mujer con que cuenta. Entre 5 y 9 trabajos aparecen en 27 publicaciones; 43 publicaciones publican 3 ó 4 trabajos, 60 tan sólo con 2 y hay 149 publicaciones que han recogido solamente 1 estudio.

En base a la similitud de los títulos de las revistas se han establecido categorías anidadas, que en un segundo nivel de reducción se han concretado en 10: Sociológicas, 
Antropológicas, Políticas, Laborales, Jurídicas, Históricas, Linguiísticas, Pedagógicas, Humanísticas y Clínicas. Como indica el análisis temático, el estudio de las mujeres compromete a todas las Ciencias Sociales, siendo objeto de reduccionismo cuando se adecua a cada especialidad. Este es uno de los principales efectos de la segmentación de las CCSS, la adaptación del objeto de estudio a la estructura parcial de cada sector de estudio, en lugar de proceder, como en su dia propiciara en la enseñanza básica la Escuela Nueva, a la adaptación de la organización científica a los problemas y objetos estudiados.

Véase en el anexo I la relación de las Revistas que componen cada grupo, así como la frecuencia con que publican artículos sobre la temática femenina.

Revisando las correlaciones significativas (siempre a un nivel $\mathrm{p}>0.000$ ) entre cada una de estas revistas especializadas y los temas en ellas publicados, encontramos la siguiente situación.

Con las revistas sociológicas se correlacionan los temas del polo social de la primera dimensión y del cuadrante inferior derecha del circumplex, es decir, los del polo solidario: Sociedad, familia, desigualdad social, justicia, obras benéficas, ayudas no oficiales, gobierno, género, matrimonio, trabajo, políticas de izquierda y acción, además de los estudios sobre personajes famosos.

En revistas antropológicas aparecen muchos estudios de temas paralelos a los que se incluyen en las de sociología, probablemente por la escasez de las últimas si se considera el volumen de investigación producido. Así los temas más publicados son demografía, estratificación, matrimonio, ambiente, empleo, profesiones, colectividades, economía, enseñanza y género. Como ocurre con las sociológicas, la inclusión de estudios sobre la mujer crece y son las preferidas por autores latinoamericanos, por universitarios y por quienes han publicado más de un estudio sobre mujeres.

Las políticas, en las que publican la mayoría de autores extranjeros, muestran una gran dispersión temática, reuniendo gran parte de los estudios sobre feminismo, economía, colectividades, políticas de izquierda, lenguaje, delincuencia y delitos sexuales.

Las laborales están altamente especializadas: estudios sobre profesión trabajo, trabajo, sectores, empleo, empresas, cooperación, asociacionismo, jurídicos (laborales), y son donde se incluyen los estudios sobre discriminación laboral así como algunos de los de ayudas. Las jurídicas también incluyen numerosos estudios sobre profesiones y coopera- 
ción, si bien se ocupan preferentemente de los temas jurídicos, de aplicación de la justicia, violencia, delincuencia, delitos y delitos sexuales.

Las históricas, preferentemente utilizadas por autores españoles, reúnen evidentemente tratados históricos sobre etapas de la historia europea, monacato, religión, personajes, iconografía, arte, literatura y fuentes de información. Son las que mayor uso hacen del descriptor mujer. Las linguíísticas por el tema en el que participan en esta base de datos son preferentemente utilizadas por autores extranjeros en estudios tanto históricos como literarios. Las pedagógicas además de temas educativos incluyen estudios sobre la historia de Europa, sobre empresas y ayudas no oficiales.

Bajo el rótulo de Humanidades se han agrupado una serie de revistas de dificil categorización, especialmente las de referencia a corrientes de pensamiento filosófico y las geográficas y en consecuencia la diversidad temática de sus publicaciones es muy amplia: arte, iconografía, literatura, lenguaje, fuentes de información, gobierno, conflicto, resistencia, historia europea, sexualidad y feminismo. Son las que muestran una mayor perdurabilidad a tenor de las elevadas cantidades que han alcanzado sus números y volúmenes y también en ellas, como en las lingüísticas, aparecen la gran mayoría de trabajos extranjeros incluídos en esta base.

En cuanto a las revistas clínicas, además de los temas que constituyen su especialidad, y que dado el elevado coeficiente de correlación entre ellos (los descriptores son estándares citados en la mayoría de los estudios) aparecen siempre como bloque, se incluyen estudios sobre los aspectos de la mujer más próximos al nivel orgánico: ovulación, fertilidad, género, pareja, deporte, sexualidad, delitos sexuales, violencia, adicciones y población, es decir, la práctica totalidad del sector individual. La inclusión de artículos referidos a mujeres aumenta con los años, lo que no es muy buen indicio si se atiende a sus contenidos patológicos. También son las revistas que, como ya se dijo en relación al bloque temático propio, incluye mayor número de descriptores metodológicos, mayor número de firmantes y autores preferentemente varones, hispanos y universitarios. No hay que olvidar que gran parte de estos autores pertecen a la profesión médica, de larga tradición científica. La correlación con las revistas más jóvenes (los números más bajos de volumen) puede significar el que estos estudios sobre sociología de la salud femenina no se incluyen en las revistas médicas de mayor tradición. A lo que hay que añadir que la gran mayoría de títulos incluídos como revistas clínicas pertenecen al sector psiquiátrico y psicoterapeútico.

Las revistas clínicas son las que más trabajos sobre la mujer publican (24.1\%), tanto del ámbito orgánico como del psiquiátrico y psicológico. Junto con las revistas especializa- 
das en humanidades (18.1\%), en Antropología (16.5\%) y en Historia (12.2.\%) han facilitado la comunicación de un $72 \%$ de las publicaciones.

La temática resume el núcleo del título de la obra. Los 855 artículos (los libros lógicamente quedan excluídos de este estudio), se han resumido en 126 temas, difíciles de concentrar en un número menor por la amplitud de matices que abarcan. En la tabla 1 se muestra la distribución de cada uno de estos temas en los 10 campos de especialización de las revistas, con un orden de frecuencias decreciente tanto en las revistas como en los temas (Chi cuadrado de 2638.185, $\mathrm{p}=0.000$ ).

Los 126 temas abordados, extraídos del núcleo fundamental del título, presentan una peligrosa dispersión de intereses y contenidos, muy alejados de la necesaria estructuración de los conocimientos que constituyen una disciplina científica. La ciencia se construye sobre modelos, leyes y teorías, que a su vez exigen mutua relación entre los elementos en que se parcializa su sector de estudio. $Y$ estas relaciones son difíciles de obtener sobre una dispersión tan inconexa como la que muestran los estudios de las mujeres.

No es que sea inadecuada la amplitud de miras ni la pluralidad de enfoques, sino que la inadecuación proviene de la ausencia de referentes comunes sobre los que puedan diseñarse los estudios y estructurarse los resultados en un cuerpo de conocimientos. El nivel de concreción de la mayoría de estos estudios impide la integración y relación entre ellos, de forma que cualquier intento de generalización con esta carencia de estructura interna, aboca en las agregaciones que tanta confusión y errores están produciendo en los diferentes campos sociológicos, con una situación semejante a la aquí analizada.

Precisamente la obtención de unos ejes comunes sobre los que estructurar esta diversidad, es uno de los objetivos de este estudio, en gran parte alcanzados, al menos con validez interna para el sector de información analizada. Queda pendiente su validación externa en otros ámbitos. 
TABLA 1. Temática investigada en las publicaciones de cada especialidad

\begin{tabular}{|c|c|c|c|c|c|c|c|c|c|c|c|c|}
\hline & \multicolumn{4}{|c|}{ Temática } & \multicolumn{6}{|c|}{ Revista } & \multirow{2}{*}{\multicolumn{2}{|c|}{ PORCENTAJE }} \\
\hline & & & & & & & & & & & & \\
\hline & Salud & Huma & Antro & Hist & Soci & Labo & Poli & Dere & Filo & Educ & $\mathrm{T}^{*}$ & $\mathbf{M}^{* *}$ \\
\hline imageMuj & 0 & 11 & 5 & 4 & 0 & 1 & 3 & 0 & 5 & 0 & 29 & 3.4 \\
\hline espacGen & 1 & 4 & 18 & 1 & 1 & 1 & 0 & 0 & 0 & 1 & 27 & 3.2 \\
\hline deporteO & 15 & 1 & 0 & 0 & 3 & 0 & 0 & 0 & 0 & 0 & 19 & 2.2 \\
\hline femEspan & 0 & 5 & 1 & 9 & 3 & 0 & 0 & 0 & 0 & 0 & 18 & 2.1 \\
\hline difGener & 9 & 3 & 1 & 0 & 3 & 2 & 0 & 0 & $\mathbf{0}$ & 0 & 18 & 2.1 \\
\hline mujObrer & 0 & 2 & 5 & 1 & 1 & 7 & 0 & 1 & 0 & 0 & 17 & 2.0 \\
\hline hispanoa & 0 & 2 & 8 & 2 & 4 & 0 & 0 & 0 & 0 & 1 & 17 & 2.0 \\
\hline terapias & 15 & 2 & 0 & 0 & 0 & 0 & 0 & 0 & 0 & 0 & 17 & 2.0 \\
\hline pensFemi & 1 & 6 & 1 & 1 & 1 & 1 & 5 & 0 & 0 & 0 & 16 & 1.9 \\
\hline prensaBi & 0 & 5 & 0 & 5 & 1 & 1 & 1 & 0 & 2 & 0 & 15 & 1.8 \\
\hline ansiedad & 15 & 0 & 0 & 0 & 0 & 0 & 0 & 0 & 0 & 0 & 15 & 1.8 \\
\hline discuMuj & 1 & 3 & 2 & 6 & 1 & 0 & 1 & 0 & 0 & 0 & 14 & 1.6 \\
\hline medioeev & 1 & 2 & 3 & 7 & 0 & 0 & 0 & 0 & 1 & 0 & 14 & 1.6 \\
\hline escriMuj & 0 & 7 & 3 & 0 & 1 & 0 & 1 & 0 & 1 & 0 & 13 & 1.5 \\
\hline prehistoria & 0 & 3 & 2 & 8 & 0 . & 0 & 0 & 0 & 0 & 0 & 13 & 1.5 \\
\hline Iconogra & 0 & 4 & 0 & 9 & 0 & 0 & 0 & 0 & 0 & 0 & 13 & 1.5 \\
\hline femEduca & 0 & 3 & 3 & 2 & 0 & 1 & 0 & 0 & 0 & 3 & 12 & 1.4 \\
\hline guerraPo & 0 & 2 & 2 & 4 & 2 & 0 & 1 & 0 & 0 & 1 & 12 & 1.4 \\
\hline tipoPers & 12 & 0 & 0 & 0 & 0 & 0 & 0 & 0 & 0 & 0 & 12 & 1.4 \\
\hline feminAgr & 0 & 1 & 9 & 0 & 1 & 0 & 1 & 0 & 0 & 0 & 12 & 1.4 \\
\hline renacimi & 0 & 4 & 3 & 2 & 1 & 0 & 0 & 1 & 0 & 0 & 11 & 1.3 \\
\hline femIdent & 6 & 2 & 1 & 0 & 1 & 0 & 1 & 0 & 0 & 0 & 11 & 1.3 \\
\hline sXIXindu & 0 & 3 & 1 & 3 & 1 & 2 & 0 & 0 & 0 & 0 & 10 & 1.2 \\
\hline femAsoci & 0 & 2 & 1 & 5 & 0 & 0 & 1 & 0 & 1 & 0 & 10 & 1.2 \\
\hline rolesMuj & 0 & 3 & 3 & 0 & 1 & 0 & 0 & 0 & 1 & 2 & 10 & 1.2 \\
\hline rolFamil & 3 & 3 & 1 & 0 & 3 & 0 & 0 & 0 & 0 & 0 & 10 & 1.2 \\
\hline esquizof & 9 & 0 & 0 & 0 & 1 & 0 & 0 & 0 & 0 & 0 & 10 & 1.2 \\
\hline salud & 9 & 0 & 0 & 0 & 0 & 0 & 0 & 0 & 0 & 0 & 9 & 1.1 \\
\hline vejez & 6 & 0 & 0 & 0 & 2 & 1 & 0 & 0 & 0 & 0 & 9 & 1.1 \\
\hline
\end{tabular}

$\mathrm{T}^{*}=$ Total

$\mathrm{M}^{* *}=$ Marginal 


\begin{tabular}{|c|c|c|c|c|c|c|c|c|c|c|c|c|}
\hline leyendas & 5 & 0 & 0 & 1 & 1 & 0 & 0 & 0 & 2 & 0 & 9 & 1.1 \\
\hline sXIX & 0 & 5 & 1 & 3 & 0 & 0 & 0 & 0 & 0 & 0 & 9 & 1.1 \\
\hline orgFemin & 0 & 4 & 3 & 0 & 0 & 0 & 1 & 0 & 0 & 0 & 8 & 0.9 \\
\hline orgPopul & 0 & 1 & 3 & 0 & 3 & 0 & 1 & 0 & 0 & 0 & 8 & 0.9 \\
\hline pornografía & 0 & 0 & 0 & 0 & 0 & 0 & 8 & 0 & 0 & 0 & 8 & 0.9 \\
\hline demografía & 0 & 0 & 7 & 0 & 0 & 1 & 0 & 0 & 0 & 0 & 8 & 0.9 \\
\hline aborto & 3 & 1 & 1 & 0 & 0 & 2 & 0 & 1 & 0 & 0 & 8 & 0.9 \\
\hline somatiza & 7 & 0 & 0 & 0 & 0 & 0 & 0 & 0 & 1 & 0 & 8 & 0.9 \\
\hline legisLab & 0 & 0 & 0 & 0 & 0 & 4 & 1 & 2 & 0 & 1 & 8 & 0.9 \\
\hline femAntig & 0 & 2 & 0 & 1 & 4 & 0 & 0 & 0 & 1 & 0 & 8 & 0.9 \\
\hline ascetismo & 0 & 5 & 0 & 2 & 1 & 0 & 0 & 0 & 0 & 0 & 8 & 0.9 \\
\hline masculina & 1 & 2 & 1 & 3 & 0 & 0 & 0 & 0 & 0 & 0 & 7 & 0.8 \\
\hline femAmeri & 0 & 1 & 1 & 1 & 2 & 0 & 2 & 0 & 0 & 0 & 7 & 0.8 \\
\hline prostitución & 0 & 2 & 3 & 2 & 0 & 0 & 0 & 0 & 0 & 0 & 7 & 0.8 \\
\hline educMuje & 1 & 1 & 0 & 1 & 0 & 0 & 0 & 0 & 0 & 4 & 7 & 0.8 \\
\hline trabDome & 1 & 2 & 2 & 0 & 1 & 0 & 0 & 1 & 0 & 0 & 7 & 0.8 \\
\hline sexuAdol & 5 & 1 & 0 & 0 & 0 & 0 & 0 & 1 & 0 & 0 & 7 & 0.8 \\
\hline ejército & 0 & 1 & 0 & 0 & 1 & 0 & 1 & 4 & 0 & 0 & 7 & 0.8 \\
\hline desiguSo & 0 & 1 & 0 & 0 & 4 & 1 & 1 & 0 & 0 & 0 & 7 & 0.8 \\
\hline discSala & 0 & 0 & 0 & 0 & 0 & 7 & 0 & 0 & 0 & 0 & 7 & 0.8 \\
\hline europeas & 0 & 1 & 1 & 0 & 0 & 0 & 5 & 0 & 0 & 0 & 7 & 0.8 \\
\hline afroAsia & 0 & 2 & 3 & 1 & 0 & 0 & 0 & 0 & 1 & 0 & 7 & 0.8 \\
\hline violación & 7 & 0 & 0 & 0 & 0 & 0 & 0 & 0 & 0 & 0 & 7 & 0.8 \\
\hline maternidad & 5 & 0 & 1 & 0 & 1 & 0 & 0 & 0 & 0 & 0 & 7 & 0.8 \\
\hline socLiter & 0 & 2 & 1 & 1 & 0 & 0 & 0 & 0 & 2 & 0 & 6 & 0.7 \\
\hline artistas & 0 & 4 & 0 & 2 & 0 & 0 & 0 & 0 & 0 & 0 & 6 & 0.7 \\
\hline empreFun & 1 & 0 & 0 & 0 & 0 & 5 & 0 & 0 & 0 & 0 & 6 & 0.7 \\
\hline migraLab & 1 & 0 & 3 & 0 & 1 & 0 & 1 & 0 & 0 & 0 & 6 & 0.7 \\
\hline violeSex & 1 & 0 & 1 & 0 & 1 & 0 & 0 & 3 & 0 & 0 & 6 & 0.7 \\
\hline prisiones & 1 & 1 & 2 & 0 & 0 & 0 & 0 & 2 & 0 & 0 & 6 & 0.7 \\
\hline menopausia & 5 & 0 & 0 & 1 & 0 & 0 & 0 & 0 & 0 & 0 & 6 & 0.7 \\
\hline socReligión & 0 & 2 & 0 & 3 & 0 & 0 & 0 & 0 & 0 & 0 & 5 & 0.6 \\
\hline oNGMovim & 0 & 1 & 2 & 0 & 2 & 0 & 0 & 0 & 0 & 0 & 5 & 0.6 \\
\hline femArte & 0 & 1 & 0 & 2 & 2 & 0 & 0 & 0 & 0 & 0 & 5 & 0.6 \\
\hline sociGene & 0 & 1 & 1 & 0 & 1 & 0 & 0 & 0 & 1 & 1 & 5 & 0.6 \\
\hline ciencMuj & 0 & 5 & 0 & 0 & 0 & 0 & 0 & 0 & 0 & 0 & 5 & 0.6 \\
\hline
\end{tabular}




\begin{tabular}{|c|c|c|c|c|c|c|c|c|c|c|c|c|}
\hline disfSexu & 5 & 0 & 0 & 0 & 0 & 0 & 0 & 0 & 0 & 0 & 5 & 0.6 \\
\hline sexuaFem & 5 & 0 & 0 & 0 & 0 & 0 & 0 & 0 & 0 & 0 & 5 & 0.6 \\
\hline comercio & 1 & 0 & 1 & 0 & 1 & 2 & 0 & 0 & 0 & 0 & 5 & 0.6 \\
\hline discSeTr & 0 & 0 & 0 & 0 & 0 & 4 & 0 & 1 & 0 & 0 & 5 & 0.6 \\
\hline barreAcc & 0 & 0 & 1 & 0 & 1 & 2 & 0 & 0 & 1 & 0 & 5 & 0.6 \\
\hline alcoholi & 5 & 0 & 0 & 0 & 0 & 0 & 0 & 0 & 0 & 0 & 5 & 0.6 \\
\hline fecundidad & 2 & 0 & 1 & 1 & 1 & 0 & 0 & 0 & 0 & 0 & 5 & 0.6 \\
\hline embarazo & 5 & 0 & 0 & 0 & 0 & 0 & 0 & 0 & 0 & 0 & 5 & 0.6 \\
\hline climater & 4 & 1 & 0 & 0 & 0 & 0 & 0 & 0 & 0 & 0 & 5 & 0.6 \\
\hline saludmen & 5 & 0 & 0 & 0 & 0 & 0 & 0 & 0 & 0 & 0 & 5 & 0.6 \\
\hline derecMuj & 1 & 2 & 0 & 0 & 0 & 1 & 0 & 1 & 0 & 0 & 5 & 0.6 \\
\hline igualOpo & 0 & 0 & 0 & 0 & 1 & 4 & 0 & 0 & 0 & 0 & 5 & 0.6 \\
\hline ptencMuj & 1 & 0 & 0 & 0 & 3 & 0 & 0 & 0 & 0 & 1 & 5 & 0.6 \\
\hline feMonaca & 0 & 1 & 0 & 3 & 0 & 0 & 0 & 0 & 0 & 0 & 4 & 0.5 \\
\hline ordenRel & 0 & 0 & 2 & 2 & 0 & 0 & 0 & 0 & 0 & 0 & 4 & 0.5 \\
\hline soColoni & 0 & 0 & 3 & 1 & 0 & 0 & 0 & 0 & 0 & 0 & 4 & 0.5 \\
\hline nivelSup & 3 & 0 & 0 & 0 & 0 & 1 & 0 & 0 & 0 & 0 & 4 & 0.5 \\
\hline modaEmpl & 0 & 0 & 1 & 0 & 0 & 3 & 0 & 0 & 0 & 0 & 4 & 0.5 \\
\hline desempEs & 0 & 0 & 1 & 0 & 0 & 3 & 0 & 0 & 0 & 0 & 4 & 0.5 \\
\hline salusegu & 0 & 0 & 1 & 0 & 0 & 2 & 1 & 0 & 0 & 0 & 4 & 0.5 \\
\hline droga & 4 & 0 & 0 & 0 & 0 & 0 & 0 & 0 & 0 & 0 & 4 & 0.5 \\
\hline delincue & 4 & 0 & 0 & 0 & 0 & 0 & 0 & 0 & 0 & 0 & 4 & 0.5 \\
\hline movFemin & 0 & 0 & 4 & 0 & 0 & 0 & 0 & 0 & 0 & 0 & 4 & 0.5 \\
\hline carrIndi & 0 & 0 & 1 & 1 & 1 & 0 & 0 & 0 & 0 & 0 & 3 & 0.4 \\
\hline mediaMuj & 1 & 1 & 0 & 0 & 0 & 0 & 0 & 0 & 1 & 0 & 3 & 0.4 \\
\hline publicidad & 1 & 1 & 0 & 0 & 0 & 0 & 0 & 0 & 1 & 0 & 3 & 0.4 \\
\hline politGen & 1 & 1 & 1 & 0 & 0 & 0 & 0 & 0 & 0 & 0 & 3 & 0.4 \\
\hline monopare & 1 & 0 & 1 & 0 & 1 & 0 & 0 & 0 & 0 & 0 & 3 & 0.4 \\
\hline econCamp & 0 & 0 & 0 & 1 & 0 & 1 & 1 & 0 & 0 & 0 & 3 & 0.4 \\
\hline homosexu & 1 & 1 & 1 & 0 & 0 & 0 & 0 & 0 & 0 & 0 & 3 & 0.4 \\
\hline español & 0 & 2 & 1 & 0 & 0 & 0 & 0 & 0 & 0 & 0 & 3 & 0.4 \\
\hline identGru & 0 & 0 & 1 & 0 & 2 & 0 & 0 & 0 & 0 & 0 & 3 & 0.4 \\
\hline depresion & 3 & 0 & 0 & 0 & 0 & 0 & 0 & 0 & 0 & 0 & 3 & 0.4 \\
\hline canceriMa & 3 & 0 & 0 & 0 & 0 & 0 & 0 & 0 & 0 & 0 & 3 & 0.4 \\
\hline derecHum & 0 & 0 & 0 & 0 & 1 & 0 & 1 & 1 & 0 & 0 & 3 & 0.4 \\
\hline derecInt & 0 & 0 & 0 & 0 & 0 & 1 & 0 & 2 & 0 & 0 & 3 & 0.4 \\
\hline
\end{tabular}




\begin{tabular}{|c|c|c|c|c|c|c|c|c|c|c|c|c|}
\hline planLabo & 0 & 0 & 0 & 0 & 1 & 2 & 0 & 0 & 0 & 0 & 3 & 0.4 \\
\hline modeDesa & 0 & 0 & 2 & 0 & 0 & 0 & 1 & 0 & 0 & 0 & 3 & 0.4 \\
\hline innoTecn & 0 & 1 & 1 & 0 & 1 & 0 & 0 & 0 & 0 & 0 & 3 & 0.4 \\
\hline progPoli & 0 & 2 & 0 & 0 & 0 & 1 & 0 & 0 & 0 & 0 & 3 & 0.4 \\
\hline poder & 0 & 1 & 0 & 0 & 1 & 1 & 0 & 0 & 0 & 0 & 3 & 0.4 \\
\hline represPo & 0 & 1 & 1 & 0 & 0 & 0 & 1 & 0 & 0 & 0 & 3 & 0.4 \\
\hline afilPolt & 0 & 2 & 1 & 0 & 0 & 0 & 0 & 0 & 0 & 0 & 3 & 0.4 \\
\hline educJesu & 0 & 0 & 1 & 0 & 0 & 0 & 1 & 0 & 0 & 0 & 2 & 0.2 \\
\hline relLiter & 0 & 1 & 0 & 1 & 0 & 0 & 0 & 0 & 0 & 0 & 2 & 0.2 \\
\hline conquAme & 0 & 0 & 1 & 0 & 1 & 0 & 0 & 0 & 0 & 0 & 2 & 0.2 \\
\hline sXX & 0 & 0 & 1 & 0 & 1 & 0 & 0 & 0 & 0 & 0 & 2 & 0.2 \\
\hline moda & 0 & 1 & 0 & 0 & 0 & 0 & 1 & 0 & 0 & 0 & 2 & 0.2 \\
\hline cultMuje & 0 & 1 & 1 & 0 & 0 & 0 & 0 & 0 & 0 & 0 & 2 & 0.2 \\
\hline violePol & .0 & 0 & 0 & 0 & 1 & 0 & 0 & 1 & 0 & 0 & 2 & 0.2 \\
\hline controNa & 2 & 0 & 0 & 0 & 0 & 0 & 0 & 0 & 0 & 0 . & 2 & 0.2 \\
\hline poliAjus & 0 & 1 & 0 & 0 & 0 & 1 & 0 & 0 & 0 & 0 & 2 & 0.2 \\
\hline orienPre & 0 & 0 & 0 & 0 & 0 & 2 & 0 & 0 & 0 & 0 & 2 & 0.2 \\
\hline policía & 0 & 0 & 0 & 0 & 1 & 0 & 0 & 1 & 0 & 0 & 2 & 0.2 \\
\hline luchaPol & 0 & 1 & 0 & 1 & 0 & 0 & 0 & 0 & 0 & 0 & 2 & 0.2 \\
\hline cambCult & 0 & 0 & 0 & 0 & 0 & 1 & 1 & 0 & 0 & 0 & 2 & 0.2 \\
\hline precolom & 0 & 1 & 0 & 0 & 0 & 0 & 0 & 0 & 0 & 0 & 1 & 0.1 \\
\hline marginac & 0 & 0 & 1 & 0 & 0 & 0 & 0 & 0 & 0 & 0 & 1 & 0.1 \\
\hline casasPop & 1 & 0 & 0 & 0 & 0 & 0 & 0 & 0 & 0 & 0 & 1 & 0.1 \\
\hline Total & 206 & 155 & 141 & 104 & 76 & 69 & 44 & 23 & 22 & 15 & 855 & \\
\hline Porcentaje & ! & & & & & & & & & & & \\
\hline Marginal & 24.1 & 18.1 & 16.5 & 12.2 & 8.9 & 8.1 & 5.1 & 2.7 & 2.6 & 1.8 & & 100.0 \\
\hline
\end{tabular}

\section{2. Época estudiada}

Puesto que en este análisis la variable de cruce tan sólo cuenta con 2 categorías, y por tanto no hay apenas dispersión de las respuestas, se ha mantenido la clasificación de revistas a un nivel más específico, por lo que las frecuencias y porcentaje marginales de la Tabla 2 ofrecen un nivel de detalle superior al de la Tabla 1. Pero téngase en cuenta, tanto en la comparación de estas dos tablas como de cualquiera otras, que los totales varían ligeramente de unas tablas a otras, por ser diferente el número de missings y de categorías 
excluídas para cada índice analizado. En el caso que nos ocupa, quedan excluídos los libros. Precisamente para favorecer las comparaciones entre tablas diferentes, todos los porcentajes se han obtenido sobre la totalidad, con lo que en parte se compensa este problema.

Con el nombre de época se construyó un índice que diferencia la publicación según sea posterior o anterior a 1950 , encontrando que un $31 \%$ de investigaciones sobre mujeres son estudios históricos, referidos a generaciones anteriores a la actual, con una fuerte dependencia de los campos humanísticos con los que la Sociología estuvo largamente integrada y por ello, más desarrollados teniendo en cuenta más los estereotipos culturales que los problemas actuales. No es este resultado un buen indicador de un campo cuyo objetivo es el análisis de la realidad social.

Además, la publicación de este $31 \%$ de estudios sobre mujeres, que es más estrictamente, sociología de la historia de las mujeres, no se distribuye aleatoriamente $\left(\mathrm{x}^{2}=355.533\right.$, $\mathrm{p}=0.000$ ), sino que se publica fundamentalmente en revistas de enfoques muy variados, pero alejado de las especialidades que definen el resto de categorías, por lo que se han incluido en la categoría de varias. La pluralidad de este conjunto de revistas se evidencia en que dedican aproximadamente el mismo espacio a estudios actuales $(7,6 \%)$ y a históri$\cos (8.4 \%)$ y según parece son las de más fácil acceso para quienes realizan estudios sobre mujeres, puesto que representan exactamente la sexta parte de las publicaciones (16.\%). Considerando que las escasas dos revistas especializadas en sociología de la mujer han publicado el $2.6 \%$ del total analizado, del que a su vez más de los dos tercios son estudios históricos, se podría concluir con la paradoja de que la especialidad viene representada por este núcleo de temática inespecífica.

Las especialidades económicas, laborales, políticas y médicas son las más dedicadas a la comunicación de estudios sobre la actualidad de las mujeres. Destacan las revistas geográficas por el gran número de publicaciones sobre la geografía del género.

Al ordenar la tabla por el total de publicaciones se muestra una clara relación entre los sectores de especialización más dedicados al estudio de las mujeres, y su orientación hacia el análisis de la situación actual. Cuando las revistas han publicado un número de trabajos inferiores al 3\%, el orden se invierte y son preferidos los estudios históricos, coincidiendo con la especialidad humanística de este bloque.

Aunque la diseminización de las publicaciones es abrumadora, con los 10 sectores de máxima productividad se acumula el $76.6 \%$ de todos los trabajos y con los 14 que publican cada uno un $2 \%$ o más, se alcanza prácticamente el $90 \%$. 
TABLA 2. Épocas estudiadas en las publicaciones de cada especialidad.

\begin{tabular}{|c|c|c|c|c|c|c|}
\hline \multirow[t]{2}{*}{$\begin{array}{l}\text { FRECUENCIAS } \\
\text { Revista }\end{array}$} & \multicolumn{3}{|c|}{$\begin{array}{l}\text { OBSERVADAS } \\
\text { Época }\end{array}$} & \multicolumn{3}{|c|}{$\begin{array}{l}\text { PORCENTAJES TOTALES } \\
\text { Época }\end{array}$} \\
\hline & actual & pasado & TOTAL & actual & pasado & TOTAL \\
\hline varias & 65 & 72 & 137 & 7.6 & 8.4 & 16.0 \\
\hline geografía & 62 & 1 & 63 & 7.3 & 0.1 & 7.4 \\
\hline general & 48 & 6 & 54 & 5.6 & 0.7 & 6.3 \\
\hline política & 43 & 10 & 53 & 5.0 & 1.2 & 6.2 \\
\hline salud & 50 & 1 & 51 & 5.8 & 0.1 & 6.0 \\
\hline aplicada & 48 & 2 & 50 & 5.6 & 0.2 & 5.8 \\
\hline americanas & 29 & 16 & 45 & 3.4 & 1.9 & 5.3 \\
\hline interven & 37 & 131 & 38 & 4.3 & 0.1 & 4.4 \\
\hline economía & 35 & 2 & 37 & 4.1 & 0.2 & 4.3 \\
\hline trabajo & 27 & 3 & 30 & 3.2 & 0.4 & 3.5 \\
\hline psiquiatría & 28 & 0 & 28 & 3.3 & 0.0 & 3.3 \\
\hline mujeres & 6 & 16 & 22 & 0.7 & 1.9 & 2.6 \\
\hline locales & 17 & 3 & 20 & 2.0 & 0.4 & 2.3 \\
\hline histgene & 8 & 12 & 20 & 0.9 & 1.4 & 2.3 \\
\hline otros & 6 & 14 & 20 & 0.7 & 1.6 & 2.3 \\
\hline filología & 6 & 10 & 16 & 0.7 & 1.2 & 1.9 \\
\hline arte & 2 & 14 & 16 & 0.2 & 1.6 & 1.9 \\
\hline jurídica & 13 & 1 & 14 & 1.5 & 0.1 & 1.6 \\
\hline filosofia & 9 & 5 & 14 & 1.1 & 0.6 & 1.6 \\
\hline histmoderna & 1 & 13 & 14 & 0.1 & 1.5 & 1.6 \\
\hline norteeste & 5 & 7. & 12 & 0.6 & 0.8 & 1.4 \\
\hline instituc & 6 & 6 & 12 & 0.7 & 0.7 & 1.4 \\
\hline pedagogía & 8 & 3 & 11 & 0.9 & 0.4 & 1.3 \\
\hline histcontemp & 1 & 9 & 10 & 0.1 & 1.1 & 1.2 \\
\hline centrosur & 2 & 7 & 9 & 0.2 & 0.8 & 1.1 \\
\hline histmedieval & 0 & 9 & 9 & 0.0 & 1.1 & 1.1 \\
\hline histantigua & 1 & 8 & 9 & 0.1 & 0.9 & 1.1 \\
\hline varios & 9 & 0 & 9 & 1.1 & 0.0 & 1.1 \\
\hline prehistoria & 0 & 5 & 5 & 0.0 & 0.6 & 0.6 \\
\hline histlocal & 0 & 5 & 5 & 0.0 & 0.6 & 0.6 \\
\hline
\end{tabular}




\begin{tabular}{|lll|l|ll|r|}
\hline humanidades & 2 & 2 & 4 & 0.2 & 0.2 & 0.5 \\
social & 3 & 1 & 4 & 0.4 & 0.1 & 0.5 \\
otras & 4 & 0 & 4 & 0.5 & 0.0 & 0.5 \\
comunicación & 2 & 0 & 2 & 0.2 & 0.0 & 0.2 \\
familia & 2 & 0 & 2 & 0.2 & 0.0 & 0.2 \\
mercado & 2 & 0 & 2 & 0.2 & 0.0 & 0.2 \\
literatura & 0 & 2 & 2 & 0.0 & 0.2 & 0.2 \\
psicoterapia & 2 & 0 & 2 & 0.2 & 0.0 & 0.2 \\
\hline TOTAL & $\mathbf{5 8 9}$ & $\mathbf{2 6 6}$ & $\mathbf{8 5 5}$ & $\mathbf{6 8 . 9}$ & $\mathbf{3 1 . 1}$ & $\mathbf{1 0 0 . 0}$ \\
\hline
\end{tabular}

\subsection{Especialidades de las revistas en las que preferentemente publica cada institu- ción investigadora.}

La Tabla 3 muestra las revistas a las que envían sus trabajos los investigadores vinculados a cada centro. Este índice tan sólo aparece en la mitad aproximada de las referencias y por tanto, la muestra queda muy reducida. Muchos de los ausentes no estarán vinculados a ningún centro de investigación concreto, pero en otros casos simplemente falta reseñarlo, por lo que han sido excluídos. Téngase este hecho en cuenta al observar los diferentes resultados en relación con las tablas anteriores.

Como en la mayoría de estudios, aparecen nítidas dependencias entre las dos variables comparadas $\left(\mathrm{x}^{2}=331.555 ; \mathrm{g} .1 .=210 ; \mathrm{p}=0.000\right)$. El $93 \%$ de las publicaciones en las que consta el centro investigador, este es o un Hospital, o la Universidad o una Institución Académica. La productividad de los otros tres centros es irrelevante, posiblemente porque se decican más a las políticas y trabajos sociales que a la investigación. La Universidad prácticamente aglutina la mayoría de estudios, lo que no resulta un buen indicador a tenor de los resultados obtenidos al analizar la temática investigada por cada institución. 
TABLA 3. Especialidades de las Revistas

en las que publica cada centro de investigación

\begin{tabular}{|c|c|c|c|c|c|c|c|c|}
\hline \multirow[t]{2}{*}{ Revista } & \multicolumn{3}{|c|}{ Centro } & \multirow[b]{2}{*}{ Oficial } & \multirow[b]{2}{*}{ InsMujer } & \multirow[b]{2}{*}{ Asociaci } & \multirow{2}{*}{ TOTAL } & \multirow{2}{*}{$\begin{array}{l}\text { PORCEN } \\
\text { TOTAL }\end{array}$} \\
\hline & Universi & InstAcad & Hospital & & & & & \\
\hline geografía & 46 & 3 & 0 & 0 & 0 & 0 & 49 & 11.2 \\
\hline general & 38 & 4 & 1 & 1 & 0 & 0 & 44 & 10.1 \\
\hline salud & 29 & 0 & 11 & 0 & 0 & 0 & 40 & 9.0 \\
\hline varias & 24 & 1 & 4 & 0 & 0 & 0 & 29 & 6.7 \\
\hline aplicada & 28 & 6 & 4 & 2 & 0 & 0 & 40 & 9.0 \\
\hline americanas & 18 & 2 & 0 & 0 & 5 & 1 & 26 & 6.0 \\
\hline psiquiatría & 16 & 0 & 8 & 1 & 0 & 0 & 25 & 5.7 \\
\hline interven & 19 & 1 & 1 & 0 & 1 & 0 & 22 & 5.0 \\
\hline economía & . 11 & 2 & 0 & 3 & 0 & 2 & 18 & 4.1 \\
\hline locales & 9 & 2 & 0 & 1 & 0 & 1 & 13 & 3.0 \\
\hline filología & 11 & 2 & 0 & 0 & 0 & 0 & 13 & 3.0 \\
\hline politíca & 8 & 2 & 0 & 1 . & 0 & 0 & 11 & 2.5 \\
\hline trabajo & 4 & 3 & 0 & 1 & 0 & 0 & 8 & 1.8 \\
\hline histgeneral & 8 & 0 & 0 & 0 & 0 & 0 & 8 & 1.8 \\
\hline histmedieval & 8 & 0 & 0 & 0 & 0 & 0 & 8 & 1.8 \\
\hline otros & 5 & 0 & 1 & 2 & 0 & 0 & 8 & 1.8 \\
\hline histmoderna & 6 & 1 & 0 & 0 & 0 & 0 & 7 & 1.6 \\
\hline arte & 6 & 1 & 0 & 0 & 0 & 0 & 7 & 1.6 \\
\hline pedagogía & 3 & 4 & 0 & 0 & 0 & 0 & 7 & 1.6 \\
\hline mujeres & 5 & 1 & 0 & 0 & 0 & 0 & 6 & 1.4 \\
\hline norteeste & 3 & 2 & 0 & 1 & 0 & 0 & 6 & 1.4 \\
\hline histcontemp & 5 & 0 & 0 & 0 & 1 & 0 & 6 & 1.4 \\
\hline humanidades & 4 & 0 & 0 & 0 & 0 & 0 & 4 & 0.9 \\
\hline filosofía & 3 & 0 & 0 & 0 & 0 & 0 & 3 & 0.7 \\
\hline jurídica & 2 & 0 & 0 & 1 & 0 & 0 & 3 & 0.7 \\
\hline centrosur & 3 & 0 & 0 & 0 & 0 & 0 & 3 & 0.7 \\
\hline instituc & 2 & 1 & 0 & 0 & 0 & 0 & 3 & 0.7 \\
\hline histantigua & 3 & 0 & 0 & 0 & 0 & 0 & 3 & 0.7 \\
\hline social & 2 & 0 & 0 & 0 & 1 & 0 & 3 & 0.7 \\
\hline otras & 1 & 0 & 0 & 2 & 0 & 0 & 3 & 0.7 \\
\hline mercado & 2 & 0 & 0 & 0 & 0 & 0 & 2 & 0.5 \\
\hline
\end{tabular}




\begin{tabular}{|lcccccccc|}
\hline literatura & 1 & 1 & 0 & 0 & 0 & 0 & 2 & 0.5 \\
familia & 1 & 0 & 0 & 0 & 0 & 0 & 1 & 0.2 \\
prehistoria & 1 & 0 & 0 & 0 & 0 & 0 & 1 & 0.2 \\
histlocal & 1 & 0 & 0 & 0 & 0 & 0 & 1 & 0.2 \\
psicoterapia & 0 & 0 & 1 & 0 & 0 & 0 & 1 & 0.2 \\
comunicación & 0 & 0 & 0 & 0 & 0 & 0 & 0 & 0.0 \\
varios & 0 & 0 & 0 & 0 & 0 & 0 & 0 & 0.0 \\
\hline TOTAL & 336 & 43 & 26 & 16 & 9 & 4 & 436 & \\
PCMARGNAIES & 77.1 & 9.9 & 6.0 & 3.7 & 2.1 & 0.9 & & 100.0 \\
\hline
\end{tabular}

\subsection{Análisis de citas de referencias}

La base de datos utilizada tan sólo aporta el número total de referencias, sin diferenciar entre referencias de citas y bibliografía sobre el tema. En otras bases se incluyen las referencias, permitiendo el análisis sociométrico o de redes de los colegios invisibles. En nuestro estudio solamente nos limitaremos a probar las posibles relaciones de este indicador en su versión más cuantitativa.

La tabla muestra que a excepción de las revistas clínicas y de sectores aplicados o muy generales, las frecuencias decrecen según aumenta el intervalo de referencias considerado $\left(\mathrm{x}^{2}=269.221 ; \mathrm{g} .1 .=148 ; \mathrm{p}=0.000\right)$. Lá distribución en cuatro grupos, además de la categoría de ninguna respuesta se ha establecido cortando por los puntos en que se han obtenido los cuartiles. De forma que exceptuando el tercio de trabajos (28\%) que no incluye referencia alguna, la mitad incluye un número igual o inferior a 16 y la otra mitad un número superior. 
Tabla 4. Frecuencias de referencias

en las publicaciones de las diferentes especialidades.

\begin{tabular}{|c|c|c|c|c|c|c|c|c|c|c|c|c|}
\hline \multicolumn{6}{|c|}{ FRECUENCIAS OBSERVADAS } & \multicolumn{7}{|c|}{$\begin{array}{c}\text { PORCENTAJES TOTALES } \\
\text { referenc }\end{array}$} \\
\hline & 0 & $1-8$ & $9-16$ & $17-28$ & $29-312$ & TOTAL & 0 & $1-8$ & $9-16$ & $17-28$ & $29-312$ & TOTAL \\
\hline varias & 56 & 25 & 22 & 18 & 16 & 137 & 6.5 & 2.9 & 2.6 & 2.1 & 1.9 & 16.0 \\
\hline geografía & 17 & 13 & 10 & 15 & 8 & 63 & 2.0 & 1.5 & 1.2 & 1.8 & 0.9 & 7.4 \\
\hline general & 6 & 2 & 6 & 19 & 21 & 54 & 0.7 & 0.2 & 0.7 & 2.2 & 2.5 & 6.3 \\
\hline política & 25 & 9 & 7 & 8 & 4 & 53 & 2.9 & 1.1 & 0.8 & 0.9 & 0.5 & 6.2 \\
\hline salud & 6 & 5 & 5 & 16 & 19 & 51 & 0.7 & 0.6 & 0.6 & 1.9 & 2.2 & 6.0 \\
\hline aplicada & 8 & 8 & 11 & 12 & 11 & 50 & 0.9 & 0.9 & 1.3 & 1.4 & 1.3 & 5.8 \\
\hline americanas & 20 & 6 & 7 & 6 & 6 & 45 & 2.3 & 0.7 & 0.8 & 0.7 & 0.7 & 5.3 \\
\hline interven & 9 & 7 & 4 & 8 & 10 & 38 & 1.1 & 0.8 & 0.5 & 0.9 & 1.2 & 4.4 \\
\hline economía & 11 & 8 & 4 & 7 & 7 & 37 & 1.3 & 0.9 & 0.5 & 0.8 & 0.8 & 4.3 \\
\hline trabajo & 17 & 5 & 1 & 2 & 6 & 31 & 2.0 & 0.6 & 0.1 & 0.2 & 0.7 & 3.6 \\
\hline psiquiatría & 6 & 3 & 7 & 4 & 8 & 28 & 0.7 & 0.4 & 0.8 & 0.5 & 0.9 & 3.3 \\
\hline mujeres & 5 & 7 & 3 & 2 & 5 & 22 & 0.6 & 0.8 & 0.4 & 0.2 & 0.6 & 2.6 \\
\hline locales & 5 & 3 & 3 & 6 & 3 & 20 & 0.6 & 0.4 & 0.4 & 0.7 & 0.4 & 2.3 \\
\hline histgeneral & 2 & 3 & 6 & 8 & 1 & 20 & 0.2 & 0.4 & 0.7 & 0.9 & 0.1 & 2.3 \\
\hline otros & 7 & 5 & 2 & 5 & 1 & 20 & 0.8 & 0.6 & 0.2 & 0.6 & 0.1 & 2.3 \\
\hline filología & 2 & 4 & 2 & 4 & 4 & 16 & 0.2 & 0.5 & 0.2 & 0.5 & 0.5 & 1.9 \\
\hline arte & 2 & 5 & 6 & 3 & 0 & 16 & 0.2 & 0.6 & 0.7 & 0.4 & 0.0 & 1.9 \\
\hline filosofía & 4 & 4 & 3 & 0 & 3 & 14 & 0.5 & 0.5 & 0.4 & 0.0 & 0.4 & 1.6 \\
\hline jurídica & 5 & 0 & 1 & 1 & 7 & 14 & 0.6 & 0.0 & 0.1 & 0.1 & 0.8 & 1.6 \\
\hline histmoder & 1 & 3 & 3 & 4 & 3 & 14 & 0.1 & 0.4 & 0.4 & 0.5 & 0.4 & 1.6 \\
\hline norteeste & 4 & 2 & 4 & 1 & 1 & 12 & 0.5 & 0.2 & 0.5 & 0.1 & 0.1 & 1.4 \\
\hline instituc & 0 & 4 & 3 & 3 & 2 & 12 & 0.0 & 0.5 & 0.4 & 0.4 & 0.2 & 1.4 \\
\hline pedagogía & 5 & 1 & 4 & 1 & 0 & 11 & 0.6 & 0.1 & 0.5 & 0.1 & 0.0 & 1.3 \\
\hline histcon & 1 & 1 & 2 & 5 & 1 & 10 & 0.1 & 0.1 & 0.2 & 0.6 & 0.1 & 1.2 \\
\hline centrosur & 1 & 2 & 3 & 2 & 1 & 9 & 0.1 & 0.2 & 0.4 & 0.2 & 0.1 & 1.1 \\
\hline histmedi & 0 & 2 & 2 & 2 & 3 & 9 & 0.0 & 0.2 & 0.2 & 0.2 & 0.4 & 1.1 \\
\hline histanti & 1 & 1 & 1 & 0 & 6 & 9 & 0.1 & 0.1 & 0.1 & 0.0 & 0.7 & 1.1 \\
\hline varios & 7 & 0 & 1 & 0 & 1 & 9 & 0.8 & 0.0 & 0.1 & 0.0 & 0.1 & 1.1 \\
\hline prehistoria & 0 & 2 & 0 & 1 & 2 & 5 & 0.0 & 0.2 & 0.0 & 0.1 & 0.2 & 0.6 \\
\hline histlocal & 2 & 0 & 1 . & 1 & 1 & 5 & 0.2 & 0.0 & 0.1 & 0.1 & 0.1 & 0.6 \\
\hline
\end{tabular}




\begin{tabular}{|lllllll|lllll|l|}
\hline humanida & 0 & 2 & 2 & 0 & 0 & 4 & 0.0 & 0.2 & 0.2 & 0.0 & 0.0 & 0.5 \\
social & 2 & 0 & 1 & 0 & 1 & 4 & 0.2 & 0.0 & 0.1 & 0.0 & 0.1 & 0.5 \\
otras & 0 & 2 & 1 & 1 & 0 & 4 & 0.0 & 0.2 & 0.1 & 0.1 & 0.0 & 0.5 \\
comunica & 1 & 0 & 1 & 0 & 0 & 2 & 0.1 & 0.0 & 0.1 & 0.0 & 0.0 & 0.2 \\
familia & 0 & 0 & 0 & 0 & 2 & 2 & 0.0 & 0.0 & 0.0 & 0.0 & 0.2 & 0.2 \\
mercado & 0 & 0 & 2 & 0 & 0 & 2 & 0.0 & 0.0 & 0.2 & 0.0 & 0.0 & 0.2 \\
literatura & 1 & 0 & 1 & 0 & 0 & 2 & 0.1 & 0.0 & 0.1 & 0.0 & 0.0 & 0.2 \\
psicoter & 0 & 1 & 1 & 0 & 0 & 2 & 0.0 & 0.1 & 0.1 & 0.0 & 0.0 & 0.2 \\
\hline TOTAL & 239 & 145 & 143 & 165 & 164 & 856 & 27.9 & 16.9 & 16.7 & 19.3 & 19.2 & 100.0 \\
\hline
\end{tabular}

\section{5. Número de páginas dedicadas a cada comunicación}

El número de páginas aparece relacionado con la orientación científica o humanística del trabajo $\left(\mathrm{x}^{2}=521.256 ; \mathrm{g} .1 .=111 ; \mathrm{p}=0.000\right)$, siendo superior en las de contenido filosófico o de pensamiento social. En cada especialidad predomina una cantidad que probablemente, se corresponde con el máximo marcado por las directrices editoriales. En general, los campos científicos con abundante producción, como es el clínico, donde es habitual publicar todos los casos que presentan algún interés, suelen tener una mayor restricción en la limitación del número de páginas, con objeto de incrementar la cantidad de publicaciones, así como de aligerar el trabajo de los comunicantes.

El hecho de que un $25 \%$ de los estudios analizados se desarrollen con una amplitud superior a las 44 páginas, no los incluye entre los más específicamente científicos. 
Tabla 5. Número de páginas por artículo habituales en cada especialidad

\begin{tabular}{|c|c|c|c|c|c|c|c|c|c|c|}
\hline \multicolumn{6}{|c|}{ FRECUENCIAS OBSERVADAS } & \multirow{2}{*}{\multicolumn{5}{|c|}{$\begin{array}{c}\text { PORCENTAJES TOTALES } \\
\text { número }\end{array}$}} \\
\hline \multirow[t]{2}{*}{ revista } & \multicolumn{5}{|c|}{ número } & & & & & \\
\hline & $1-6$ & $7-17$ & $18-43$ & $44-574$ & TOTAL & $1-6$ & $7-17$ & $18-43$ & $44-574$ & TOTAL \\
\hline varias & 24 & 20 & 36 & 53 & 133 & 2.9 & 2.5 & 4.4 & 6.5 & 16.3 \\
\hline geografía & 7 & 7 & 43 & 5 & 62 & 0.9 & 0.9 & 5.3 & 0.6 & 7.6 \\
\hline general & 23 & 7 & 7 & 16 & 53 & 2.8 & 0.9 & 0.9 & 2.0 & 6.5 \\
\hline política & 7 & 32 & 9 & 5 & 53 & 0.9 & 3.9 & 1.1 & 0.6 & 6.5 \\
\hline aplicada & 26 & 6 & 9 & 9. & 50 & 3.2 & 0.7 & 1.1 & 1.1 & 6.1 \\
\hline salud & 15 & 1 & 26 & 5 & 47 & 1.8 & 0.1 & 3.2 & 0.6 & 5.8 \\
\hline americanas & 7 & 10 & 18 & 10 & 45 & 0.9 & 1.2 & 2.2 & 1.2 & 5.5 \\
\hline interven & 4 & 12 & 2 & 20 & 38 & 0.5 & 1.5 & 0.2 & 2.5 & 4.7 \\
\hline economía & 6 & 25 & 6 & 0 & 37 & 0.7 & 3.1 & 0.7 & 0.0 & 4.5 \\
\hline trabajo & 4 & 12 . & 10 & 5 & 31 & 0.5 & 1.5 & 1.2 & 0.6 & 3.8 \\
\hline psiquiatría & 8 & 0 & 0 & 16 & 24 & 1.0 & 0.0 & 0.0 & 2.0 & 2.9 \\
\hline mujeres & 22 & 0 & 0 & 0 & 22 & 2.7 & 0.0 & 0.0 & 0.0 & 2.7 \\
\hline locales & 0 & 8 & 6 & 5 & 19 & 0.0 & 1.0 & 0.7 & 0.6 & 2.3 \\
\hline otros & 3 & 5 & 3 & 8 & 19 & 0.4 & 0.6 & 0.4 & 1.0 & 2.3 \\
\hline histgeneral & 3 & 6 & 2 & 8 & 19 & 0.4 & 0.7 & 0.2 & 1.0 & 2.3 \\
\hline arte & 4 & 7 & 1 & 4 & 16 & 0.5 & 0.9 & 0.1 & 0.5 & 2.0 \\
\hline filosofía & 0 & 1 & 0 & 13 & 14 & 0.0 & 0.1 & 0.0 & 1.6 & 1.7 \\
\hline jurídica & 2 & 2 & 2 & 8 & 14 & 0.2 & 0.2 & 0.2 & 1.0 & 1.7 \\
\hline histmoderna & 2 & 10 & 2 & 0 & 14 & 0.2 & 1.2 & 0.2 & 0.0 & 1.7 \\
\hline norteeste & 4 & 2 & 1 & 5 & 12 & 0.5 & 0.2 & 0.1 & 0.6 & 1.5 \\
\hline pedagogía & 1 & 1 & 4 & 5 & 11 & 0.1 & 0.1 & 0.5 & 0.6 & 1.3 \\
\hline filología & 5 & 3 & 2 & 0 & 10 & 0.6 & 0.4 & 0.2 & 0.0 & 1.2 \\
\hline histcont & 4 & 4 & 2 & 0 & 10 & 0.5 & 0.5 & 0.2 & 0.0 & 1.2 \\
\hline varios & 2 & 0 & 5 & 1 & 8 & 0.2 & 0.0 & 0.6 & 0.1 & 1.0 \\
\hline centrosur & 2 & 2 & 3 & 1 & 8 & 0.2 & 0.2 & 0.4 & 0.1 & 1.0 \\
\hline histmedi & 1 & 2 & 4 & 0 & 7 & 0.1 & 0.2 & 0.5 & 0.0 & 0.9 \\
\hline instituc & 1 & 3 & 2 & 0 & 6 & 0.1 & 0.4 & 0.2 & 0.0 & 0.7 \\
\hline prehistoria & 2 & 3 & 0 & 0 & 5 & 0.2 & 0.4 & 0.0 & 0.0 & 0.6 \\
\hline histlocal & 0 & 2 & 3 & 0 & 5 & 0.0 & 0.2 & 0.4 & 0.0 & 0.6 \\
\hline humanida & 3 & 1 & 0 & 0 & 4 & 0.4 & 0.1 & 0.0 & 0.0 & 0.5 \\
\hline otras & 2 & 0 & 0 & 2 & 4 & 0.2 & 0.0 & 0.0 & 0.2 & 0.5 \\
\hline
\end{tabular}




\begin{tabular}{|lcccc|c|cccc|c|}
\hline social & 1 & 3 & 0 & 0 & 4 & 0.1 & 0.4 & 0.0 & 0.0 & 0.5 \\
comunica & 2 & 0 & 0 & 0 & 2 & 0.2 & 0.0 & 0.0 & 0.0 & 0.2 \\
familia & 2 & 0 & 0 & 0 & 2 & 0.2 & 0.0 & 0.0 & 0.0 & 0.2 \\
mercado & 0 & 1 & 0 & 1 & 2 & 0.0 & 0.1 & 0.0 & 0.1 & 0.2 \\
literatura & 2 & 0 & 0 & 0 & 2 & 0.2 & 0.0 & 0.0 & 0.0 & 0.2 \\
histantigua & 2 & 0 & 0 & 0 & 2 & 0.2 & 0.0 & 0.0 & 0.0 & 0.2 \\
psicoter & 0 & 1 & 1 & 0 & 2 & 0.0 & 0.1 & 0.1 & 0.0 & 0.2 \\
criminol & 0 & 0 & 0 & 0 & 0 & 0.0 & 0.0 & 0.0 & 0.0 & 0.0 \\
& & & & & & & & & & \\
TOTAL & 203 & 199 & 209 & 205 & 816 & 24.9 & 24.4 & 25.6 & 25.1 & 100.0 \\
\hline
\end{tabular}

El análisis de correspondencias entre el número de páginas, clasificado en 4 intervalos a partir de los cuartiles y la especialidad de las revistas ofrece el mapa mostrado a: continuación, en el que ambos ejes explican una cantidad equivalente de inercia $(37.5 \%$ el primero y $32.8 \%$ el segundo), sumando un total del $70.3 \%$, cantidad suficiente dado el amplio número de especialidades incluídas.

En la fig. 2 los números de páginas se distribuyen en cada cuadrante, y junto a ellas las especialidades asociadas con cada cantidad, que se oponen en cada diagonal.

Fig. 2. Gráfico de correspondencias entre número de páginas del artículo y especialidad de la revista

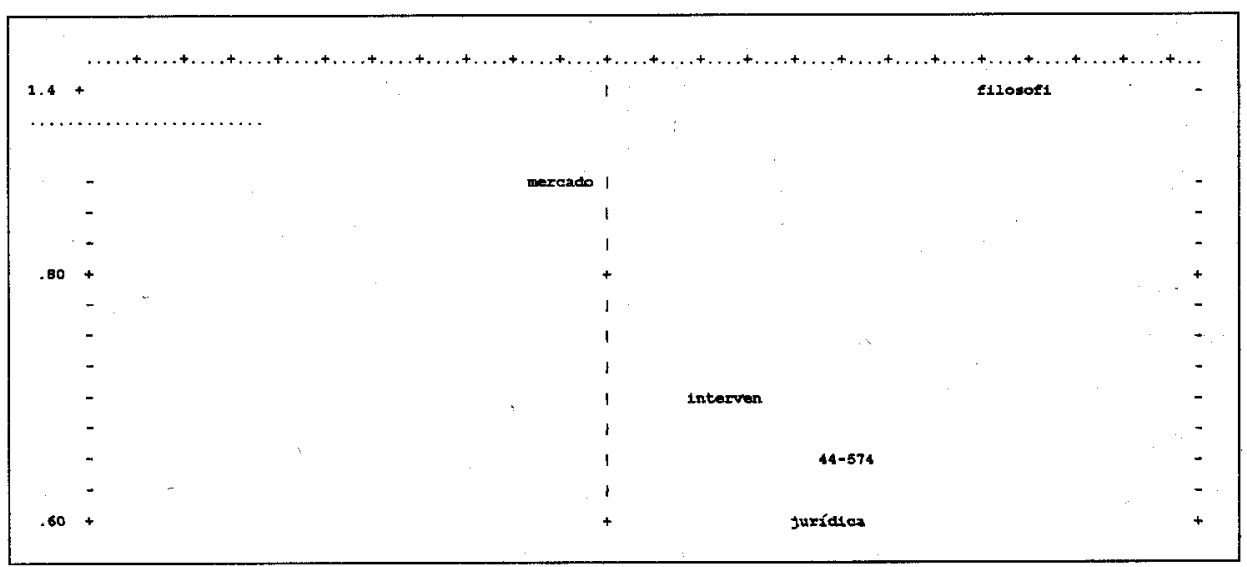


Fig. 2. Gráfico de correspondencias entre número de páginas del artículo y especialidad de la revista

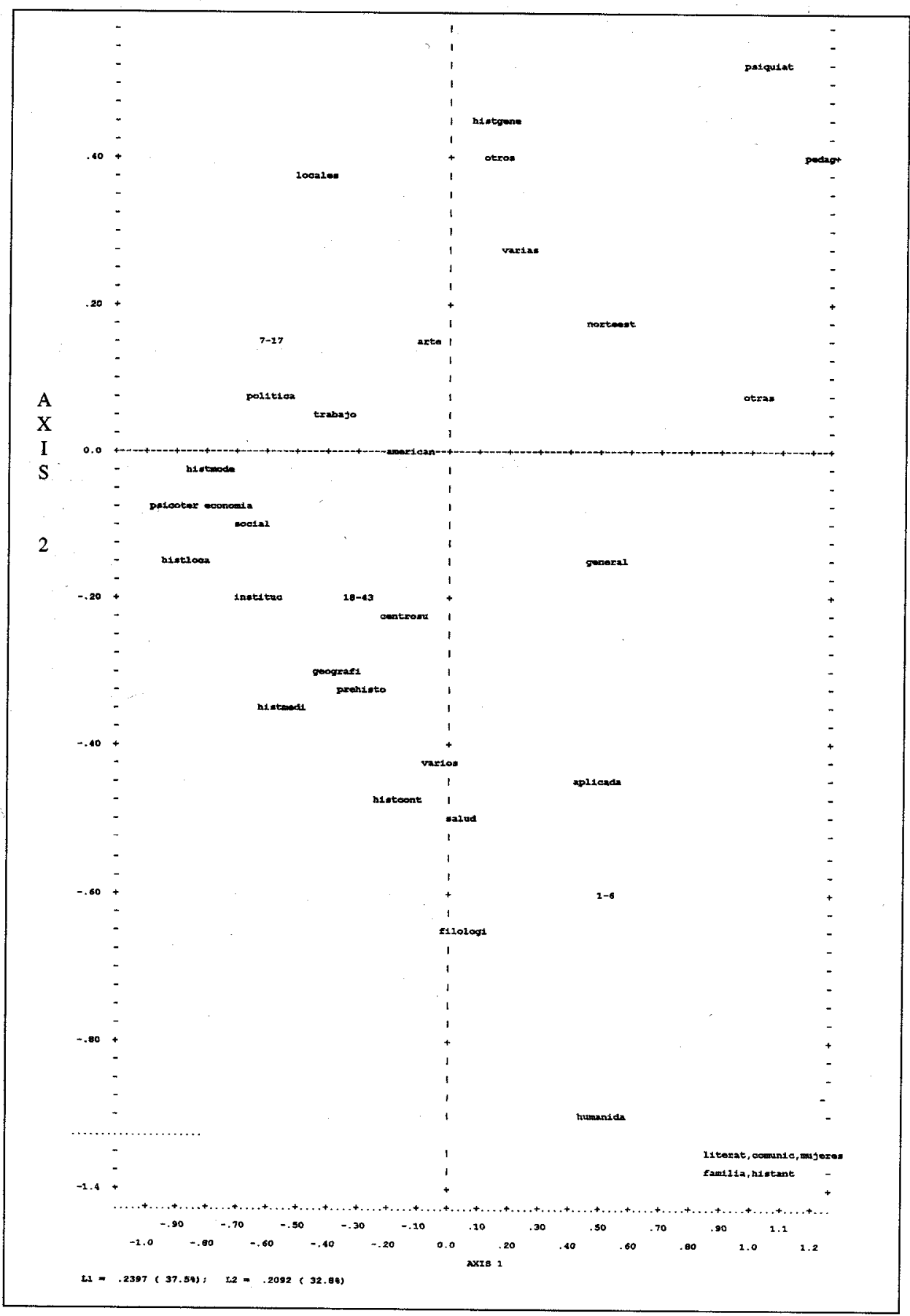




\section{6. Número de firmantes}

El número de firmantes de los trabajos aparece claramente diferenciado en relación a la especialidad en que se publica $\left(x^{2}=416.007\right.$; $\left.\mathrm{g} .1=222 ; \mathrm{p}=0.000\right)$ en el mismo sentido de los demás indicadores de nivel científico alcanzado por la especialidad. El sector clínico y aplicado, así como el más general, es al que se dedican la mayoría de equipos de investigación. No son habituales estudios en que participen más de tres autores (4\%) y aún los equipos de tres personas son poco frecuentes (6.1\%). Tampoco son habituales los trabajos firmados por colectivos ( $1.5 \%$ ), lo que indica la escasa producción científica de las múltiples asociaciones de mujeres.

Tabla 6.- Número de firmantes en cada especialidad de las revistas

\begin{tabular}{|c|c|c|c|c|c|c|c|c|}
\hline \multirow[t]{2}{*}{ revista } & \multicolumn{5}{|c|}{ firmante } & \multirow[b]{2}{*}{ colectivos } & \multirow[b]{2}{*}{ TOTAL } & \multirow[b]{2}{*}{ PORCEN } \\
\hline & unautor & pareja & tres & cuatro & cinco & & & \\
\hline varias & 118 & 10 & 2 & 0 & 0 & 0 & 130 & 15.6 \\
\hline general & 23 & 18 & 10 & 0 & 1 & 1 & 53 & 6.3 \\
\hline política & 47 & 3 & 0 & 0 & 1 & 0 & 51 & 6.1 \\
\hline economía & 29 & 4 & 1 & 0 & 0 & 0 & 34 & 4.1 \\
\hline trabajo & 27 & 3 & 1 & 0 & 0 & 0 & 31 & 3.7 \\
\hline geografía & 53 & 9 & 1 & 0 & 0 & 0 & 63 & 7.5 \\
\hline salud & 17 & 13 & 9 & 3 & 3 & 6 & 51 & 6.1 \\
\hline aplicada & 26 & 13 & 7 & 0 & 2 & 2 & 50 & 6.0 \\
\hline americanas & 40 & 0 & 1 & 0 & 0 & 0 & 41 & 4.9 \\
\hline intervención & 20 & 6 & 8 & 4 & 0 & $\mathbf{0}$ & 38 & 4.6 \\
\hline psiquiatría & 5 & 6 & 7 & 5 & 1 & 4 & 28 & 3.4 \\
\hline mujeres & 22 & 0 & 0 & 0 & 0 & 0 & 22 & 2.6 \\
\hline locales & 14 & 4 & 2 & 0 & 0 & 0 & 20 & 2.4 \\
\hline histgeneral & 17 & 3 & 0 & 0 & 0 & 0 & 20 & 2.4 \\
\hline otros & 20 & 0 & 0 & 0 & 0 & 0 & 20 & 2.4 \\
\hline filología & 14 & 2 & 0 & 0 & 0 & 0 & 16 & 1.9 \\
\hline arte & 15 & 0 & 1 & 0 & 0 & 0 & 16 & 1.9 \\
\hline filosofía & 13 & 1 & 0 & 0 & 0 & 0 & 14 & 1.7 \\
\hline jurídica & 12 & 2 & 0 & 0 & 0 & 0 & 14 & 1.7 \\
\hline histmoderna & 11 & 3 & 0 & 0 & 0 & 0 & 14 & 1.7 \\
\hline norteeste & 11 & 0 & 1 & 0 & 0 & 0 & 12 & 1.4 \\
\hline
\end{tabular}




\begin{tabular}{|lcccccc|c|c|}
\hline instituc & 11 & 1 & 0 & 0 & 0 & 0 & 12 & 1.4 \\
pedagogía & 7 & 4 & 0 & 0 & 0 & 0 & 11 & 1.3 \\
histcon & 8 & 2 & 0 & 0 & 0 & 0 & 10 & 1.2 \\
centrosur & 8 & 1 & 0 & 0 & 0 & 0 & 9 & 1.1 \\
histmedieval & 9 & 0 & 0 & 0 & 0 & 0 & 9 & 1.1 \\
histantigua & 9 & 0 & 0 & 0 & 0 & 0 & 9 & 1.1 \\
varios & 7 & 0 & 0 & 0 & 0 & 0 & 7 & 0.8 \\
histlocal & 5 & 0 & 0 & 0 & 0 & 0 & 5 & 0.6 \\
humanidades & 4 & 0 & 0 & 0 & 0 & 0 & 4 & 0.5 \\
prehistoria & 4 & 0 & 0 & 0 & 0 & 0 & 4 & 0.5 \\
otras & 3 & 1 & 0 & 0 & 0 & 0 & 4 & 0.5 \\
social & 1 & 2 & 0 & 0 & 0 & 0 & 3 & 0.4 \\
comunicación & 1 & 1 & 0 & 0 & 0 & 0 & 2 & 0.2 \\
familia & 1 & 1 & 0 & 0 & 0 & 0 & 2 & 0.2 \\
mercado & 1 & 1 & 0 & 0 & 0 & 0 & 2 & 0.2 \\
literatura & 2 & 0 & 0 & 0 & 0 & 0 & 2 & 0.2 \\
psicoterapia & 2 & 0 & 0 & 0 & 0 & 0 & 2 & 0.2 \\
\hline TOTAL & 637 & 114 & 51 & 12 & 8 & 13 & 835 & \\
PORCENTAJES & 76.3 & 13.7 & 6.1 & 1.4 & 1.0 & 1.5 & & 100.0 \\
\hline
\end{tabular}

\subsection{Productividad del investigador en relación con la especialidad de las revistas en que publica}

Como detecta el análisis de la evolución histórica, la publicación de estudios sobre las mujeres no adquiere relevancia hasta finales de los 80 , es por tanto en una escasa década cuando se han realizado casi todos los estudios, por ello es difícil encontrar especialistas con tres o más obras publicadas (7\%), limitadas a las especialidades clínicas y a los estudios de geografía del género. Sí son más frecuentes los autores con dos obras. La relación entre las dos dimensiones comparadas aparece con la misma significación que en el resto de indicadores ( $\mathrm{x}^{2}=157.397 ; \mathrm{g} .1 .=74 ; \mathrm{p}=0.000$ ), resultados que los valida como medidas equivalentes de la diferente orientación científica que tienen las revistas según su especialidad. 
Esta tabla y la anterior, son asimismo un claro exponente de que la investigación de la mujer, al menos hasta el momento, no puede considerarse una línea de investigación especialmente vinculada a equipos que la lideren, salvo los que aparecen en los estudios clínicos, que dada su parcialidad de enfoque y hasta oposición con la perspectiva sociológica, no son los más representativos de la investigación en sociología del género. No obstante, conviene integrar sus hallazgos, por la importancia que sus estudios pueden aportar respecto a las consecuencias orgánicas y psíquicas de determinados problemas sociales. Integración que exige cierto grado de interdisciplinaridad.

El perfil de este análisis dista mucho del obtenido en la mayoría de campos científicos, donde sobre un escaso número de autores extraordinariamente productivos, se van diversificando los equipos de investigación. En la sociología de la mujer, los focos de investigación están excesivamente incomunicados y son muy diversos y numerosos. Alguna intervención para conseguir una mayor concentración de esfuerzos sería necesaria para erigir una auntentica disciplina científica. Además los estudios, tal como se presentan, están más directamente dirigidos a la descripción de los problemas, que a su tratamiento como materia de análisis científico. Y aunque el enfoque más objetivo no tenga una repercusión inmediata sobre los problemas, no hay que olvidar que los cambios de estereotipos y actitudes, los cambios culturales que la mayoría de los temas abordados en las publicaciones requieren, tan sólo pueden afrontarse desde instancias que superen las situaciones concretas y que, como en el caso de las teorías y descubrimientos científicos, adquieran suficiente autoridad para que sean transmitidos como normas socializantes.

\section{Tabla 7. Productividad del investigador en relación con la especialidad de las revistas en que publica}

\begin{tabular}{|c|c|c|c|c|c|c|c|c|}
\hline \multicolumn{4}{|c|}{ FRECUENCIAS OBSERVADAS } & \multirow{2}{*}{\multicolumn{4}{|c|}{$\begin{array}{c}\text { PORCENTAJES TOTALES } \\
\text { obras }\end{array}$}} & \multirow[b]{3}{*}{ TOTAL } \\
\hline \multirow[t]{2}{*}{ revista } & \multicolumn{3}{|c|}{ obras } & & & & & \\
\hline & única & repetida & tres $0+$ & TOTAL & única & repetida & tres o+ & \\
\hline varias & 114 & 19 & 1 & 134 & 13.4 & 2.2 & 0.1 & 15.8 \\
\hline geografía & 31 & 16 & 16 & 63 & 3.7 & 1.9 & 1.9 & 7.4 \\
\hline general & 39 & 10 & 4 & 53 & 4.6 & 1.2 & 0.5 & 6.2 \\
\hline política & 46 & 6 & 0 & 52 & 5.4 . & 0.7 & 0.0 & 6.1 \\
\hline salud & 36 & 11 & 4 & 51 & 4.2 & 1.3 & 0.5 & 6.0 \\
\hline aplicada & 36 & 14 & 0 & 50 & 4.2 & 1.7 & 0.0 & 5.9 \\
\hline americanas & 33 & 7 & 3 & 43 & 3.9 & 0.8 & 0.4 & 5.1 \\
\hline
\end{tabular}




\begin{tabular}{|c|c|c|c|c|c|c|c|c|}
\hline intervención & 21 & 7 & 10 & 38 & 2.5 & 0.8 & 1.2 & 4.5 \\
\hline economía & 27 & 8 & 2 & 37 & 3.2 & 0.9 & 0.2 & 4.4 \\
\hline trabajo & 28 & 3 & 0 & 31 & 3.3 & 0.4 & 0.0 & 3.7 \\
\hline psiquiatría & 14 & 8 & 6 & 28 & 1.7 & 0.9 & 0.7 & 3.3 \\
\hline mujeres & 15 & 5 & 2 & 22 & 1.8 & 0.6 & 0.2 & 2.6 \\
\hline locales & 15 & 4 & 1 & 20 & 1.8 & 0.5 & 0.1 & 2.4 \\
\hline histgeneral & 16 & 3 & 1 & 20 & 1.9 & 0.4 & 0.1 & 2.4 \\
\hline otros & 18 & 1 & 1 & 20 & 2.1 & 0.1 & 0.1 & 2.4 \\
\hline filología & 16 & 0 & 0 & 16 & 1.9 & 0.0 & 0.0 & 1.9 \\
\hline arte & 15 & 1 & 0 & 16 & 1.8 & 0.1 & 0.0 & 1.9 \\
\hline filosofía & 12 & 2 & 0 & 14 & 1.4 & 0.2 & 0.0 & 1.7 \\
\hline jurídica & 9 & 1 & 4 & 14 & 1.1 & 0.1 & 0.5 & 1.7 \\
\hline histmoderna & 10 & 4 & 0 & 14 & 1.2 & 0.5 & 0.0 & 1.7 \\
\hline norteeste & 12 & 0 & 0 & 12 & 1.4 & 0.0 & 0.0 & 1.4 \\
\hline instituc & 8 & 4 & 0 & 12 & 0.9 & 0.5 & 0.0 & 1.4 \\
\hline pedagogía & 9 & 1 & 1 & 11 & 1.1 & 0.1 & 0.1 & 1.3 \\
\hline histcon & 8 & 2 & 0 & 10 & 0.9 & 0.2 & 0.0 & 1.2 \\
\hline centrosur & 6 & 1 & 2 & 9 & 0.7 & 0.1 & 0.2 & 1.1 \\
\hline histmedieval & 6 & 3 & 0 & 9 & 0.7 & 0.4 & 0.0 & 1.1 \\
\hline histantigua & 4 & 3 & 2 & 9 & 0.5 & 0.4 & 0.2 & 1.1 \\
\hline varios & 9 & 0 & 0 & 9 & 1.1 & 0.0 & 0.0 & 1.1 \\
\hline histlocal & 3 & 2 & 0 & 5 & 0.4 & 0.2 & 0.0 & 0.6 \\
\hline humanidades & 4 & 0 & 0 & 4 & 0.5 & 0.0 & 0.0 & 0.5 \\
\hline prehistoria & 4 & 0 & 0 & 4 & 0.5 & 0.0 & 0.0 & 0.5 \\
\hline social & 3 & 1 & 0 & 4 & 0.4 & 0.1 & 0.0 & 0.5 \\
\hline otras & 3 & 0 & 1 & 4 & 0.4 & 0.0 & 0.1 & 0.5 \\
\hline comunicación & 2 & 0 & 0 & 2 & 0.2 & 0.0 & 0.0 & 0.2 \\
\hline familia & 1 & 1 & 0 & 2 & 0.1 & 0.1 & 0.0 & 0.2 \\
\hline mercado & 2 & 0 & 0 & 2 & 0.2 & 0.0 & 0.0 & 0.2 \\
\hline literatura & 2 & 0 & 0 & 2 & 0.2 & 0.0 & 0.0 & 0.2 \\
\hline psicoterapia & 2 & 0 & 0 & 2 & 0.2 & 0.0 & 0.0 & 0.2 \\
\hline TOTAL & 639 & 148 & 61 & 848 & 75.4 & 17.5 & 7.2 & 100.0 \\
\hline
\end{tabular}




\subsection{Afianzamiento de las revistas}

Se ha utilizado el número de la revista o publicación y el volumen como indicador, detectándose que los estudios sobre las mujeres se publican preferentemente en las de reciente creación. Uno y otro indicador, aunque mantienen entre sí una elevada correlación (0.80) no coinciden porque en algunas publicaciones el volumen sigue un orden creciente pero el número repite ciclos para el año o para el volumen. Para un uso más eficiente de este indicador, las bases de datos deberían aportar información adicional acerca de las revistas incluídas, como su periodicidad, fecha de inicio y sistema de denominación para poder obtener equivalencias entre números y volúmenes.

Además este resultado debe interpretarse en relación a las proporciones totales de revistas de nueva creación en los campos de especialidad estudiados. A falta de este referente, los datos absolutos parecen indicar o bien una relación de este tema con autores y editores jóvenes (las bases de datos tampoco aportan la edad de los autores), o bien el abandono del estudio de las mujeres por parte de quienes publican en las revistas más afianzadas históricamente y más insertadas entre los científicos de su especialidad.

Las investigaciones publicadas desde Universidades son las que aparecen en los números y volúmenes más nuevos, y también los estudios sobre poblaciones españolas y psicología, probablemente por la reciente iniciación en nuestro país de gran número de revistas sobre esta disciplina, siempre editadas desde círculos universitarios. A su vez las que aparecen en los números más altos son las de humanidades, literatura y lenguaje y aquellas que aparecen en los volúmenes de número más elevado son también las de humanidades y las de aspectos antropométricos, así como las publicadas en el extranjero y las que incluyen descriptores metodológicos, hecho que parece indicar una mayor exigencia de rigor científico para las publicaciones que se mantienen durante más tiempo.

La época del estudio aparece bastante independiente del número y volumen, aunque alcanzan significación estadística para el número $\left(\mathrm{x}^{2}=12.912 ; \mathrm{g} . \mathrm{l} .=3 ; \mathrm{p}=0.000\right)$ así como para el volumen $\left(\mathrm{x}^{2}=12.700 ; \mathrm{g} .1 .=3, \mathrm{p}=0.000\right)$, debido a que las revistas más antiguas tienden a incluir investigaciones sobre hechos actuales. 


\section{REFERENCIAS}

- ARCE, C. (1993) Escalamiento multidimensional, Barcelona, PPU.

- ARCE, C. (1994) Técnicas de construcción de escalas psicológicas, Madrid, Síntesis.

- BMDP/DYNAMIC (1993): BMDP Stadistical Software Inc, Ireland, Cork. Technologogy Park, Model Farm Rd.

- CARTTER, A.M. (1966): An assessment of quality in graduate education, Washington DC., American Council on Education.

- COLE, J.A. y COLE, S. (1973): Social stratification in science, Chicago, Univ. of Chicago Press.

- CRANE, D. (1972): Invisible colleges. Diffusion of knowledge in scientific communities, Chicago, The University of Chicago Press.

- GARDFIELD E., MALIN N.V. y SMALL, H. (1978): Citation data as Science Indicators: Toward a Metric of Science, N.Y., J. Wiley.

- GIFFORD, R. y O'CONNOR, B. (1987): «The Interpersonal Circumplex as a Behavior Map», J. of Pers. and Social Psych., 52 (5): 1019-1026.

- HUBER, J.J. (1977): «Bibliometric models for journal productivity», Social Indicators research, 4: 441-473.

- JONES, L.V., LINDZEY G. y COGGESHAL, P.E. (1982): An assessment of researchdoctorate programs in the Unived States: Social Sciences, Washington, DC, National Academy Press.

- KRUSKAL, J.B. (1964a): «Multidimensional scaling by optimizing goodness of fit to a nonmetric hypothesis», Psychometrika, 29: 1-27.

- KRUSKAL, J.B. (1964b): «Nonmetric multidimensional scaling: A numerical method». Psychometrika, 29: 115-129.

- LAZARSFELD, P. y THIELENS, W. (1958): The Academic mind, Free Press, Glencoe, Ill.,

- MERTON, R.K. (1973): The sociology of science. Theoretical and empirical investigation, The University of Chicago Press, Chicago.

- PLATZ,A. (1965): «Lotka's law and research visibility», Psychological Report, 16: $566-568$

- PRICE, D.J.S. (1961): Science since Babylon. New Have Conn, Yale Univ. Press. 
- RIVAS, F., CALATAYUD, R. y PERIS, M.D. (1985): «La investigación experimental y diferencial en CCEE a través de los Congresos de Pedagogía $(1968,1972,1976) »$, Bordón, 259: 571-595.

- ROOSE, K.D. y Anderson, C.J. (1970): A rating of graduate programs, Washington DC, American Council on Education.

- SCIENCE CITATION INDEX. (1964): Philadelphia, Institute for Scientific Information.

- SHYE, S. (ed.) (1978): Theory construction and data analysis in the behavioral sciences, Ca., S.Fco, Jossey Bass.

- SIMON, H.(1971): El comportamiento administrativo, Madrid, Aguilar.(orig. 1954)

- SOCIAL SCIENCE CITATION INDEX (1974): Philadelphia, Institute for Scientificic Information.

- SPSS (1997): SPSS para Windows, Chicago, SPSS Inc.

- STRONG, S.R. y HILLS, H.I. (1986): Interpersonal comunnication Rating Scale, Richmond, VA, Virginia, Commonwealth University.

- ZIMAN,J. (1968): Public Knowledge. The social dimension of science, Cambridge, University Press.

- ZUCKERMAN, H.A. y MERTON, R.K. (1971): «Patterns of evaluation in science: Institutionalism structure and functions of the refered systems», Minerva, 9 (1): 66-100. 


\section{ANEXO I \\ CLASIFICACIONES ANIDADAS DE PRIMER Y SEGUNDO NIVEL DE LAS REVISTAS}

Para facilitar el uso más conveniente en cada análisis de uno u otro nivel de especialización, el primer dígito se refiere al nivel más general y la combinación del primero y segundo al más concreto. Un tercer dígito incluye la posibilidad de realizar análisis para revistas concretas.

Entre paréntesis figura el número de títulos distintos incluidos en cada categoría.

\section{Sociología}

10. general

11. locales

12. comunicación

13. mujeres

14. familia

15. otras

16. trabajo/política social

\section{Laboral - Empresarial}

21. economía

22. trabajo

23. mercado

\section{Derecho}

31. varios

32. criminología

\section{Política}

\section{Geografía - Antropología}

51. geografia general

52. locales americanos

53. locales centro-sur

54. locales norte-este

55. instituciones/admción 


\section{Filolología - Literatura}

61. filologia

62. literatura

63. otros

\section{Historia}

70. historia general

71. historia contemporánea

72. historia moderna

73. historia medieval

74. historia antigua

75. prehistoria, arqueología

76. historia local

77. arte

78. otros

\section{Educación}

80. pedagógica

81. social

9. Psicología - Psiquiatría - Salud

91. general

92. aplicada

93. terapias/intervención

94. clínica/salud

95. psiquiatria

\section{Humanidades - Pensamiento - Filosofia}

01. humanidades

02. filosofía

03-8. multidisciplinares 


\section{ANEXO II \\ FRECUENCIAS DE ARTICULOS PUBLICADOS EN CADA REVISTA}

El valor de la izquierda indica la frecuencia decreciente de estudios sobre la mujer publicados en cada una de las revistas, cuyos nombres aparecen ordenados alfabéticamente dentro de cada frecuencia.

41 DOCUMENTS D'ANALISI GEOGRAFICA

25 PODER Y LIBERTAD

24 CUADERNOS DE MEDICINA PSICOSOMATICA

23 ECONOMIA Y SOCIOLOGIA DEL TRABAJO

19 ANALISIS Y MODIFICACION DE CONDUCTA

16 INFORMACIONES PSIQUIATRICAS

14 AFRICA AMERICA LATINA CUADERNOS

13 DUODA

12 ASPARKIA. INVESTIGACIO FEMINISTA

12 CANELOBRE

12 INFORMACIO PSICOLOGICA

12 MON LABORAL

12 REVISTA DE PSICOLOGIA DEL DEPORTE

11 PSICOTHEMA

10 ARENAL. REVISTA DE HISTORIA DE LAS MUJERES

10 NOUS HORITZONS

9 APERTURA. CUADERNOS DE PSICOANALISIS

9 CLINICA Y SALUD

9 MIENTRAS TANTO

9 REALES SITIOS

8 ARBOR

7 AMERICA LATINA, HOY. REVISTA DE IENCIAS SOCIALES

7 ANALES DE GEOGRAFIA DE LA UNIVERSIDADCOMPLUTENSE

7 DOCUMENTACION SOCIAL

7 FOLIA NEUROPSЮUIATRICA DEL SUR Y ESTE DE ESPAÑA

7 HISTORIA 16

6 INDIGENISMO. BOLETIN SEM. ESP. ESTUDIOS INDIGENISTAS

6 REVISTA DE PSICOLOGIA SOCIAL

6 REVISTA DE PSICOLOGIA SOCIAL APLICADA

6 REVISTA ESPAÑOLA DE INVESTIGACIONES SOCIOLOGICAS 
5 ANUARIO DE ESTUDIOS AMERICANOS

5 AVENC, L'

5 BOLETIN AMERICANISTA

5 CAPITAL HUMANO

5 CIENCIA POLICIAL

5 CUADERNOS DE ESTUDIOS MEDIEVALES Y CIENCIAS Y TECNICAS HISTORIOGRAFICAS

5 ENCUENTROS EN PSICOLOGIA

5 RELACIONES LABORALES. REVISTA CRITICA DE TEORIA Y PRACTICA

5 REVISTA DE DIALECTOLOGIA Y TRADICIONES POPULARES

5 REVISTA DE OCCIDENTE

5 REVISTA DE PSICOLOGIA DE LA SALUD

5 REVISTA DE PSICOLOGIA GENERAL Y APLICADA

5 REVISTA IBEROAMERICANA DE AUTOGESTION Y ACCION COMUNAL

4 APUNTES DE PSICOLOGIA

4 CIVITAS. REVISTA ESPAÑOLA DE DERECHO DEL TRABAJO

4 CLAVES DE RAZON PRACTICA

4 CODEX AQUILARENSIS

4 COMUNICACION PSIQUIATRICA

4 CUADERNOS DE TRABAJO SOCIAL

4 CUADERNOS HISPANOAMERICANOS

4 GERION

4 HISTORIA Y FUENTE ORAL

4 PAPELES PARA LA PAZ

4 POLITICA Y SOCIEDAD

4 PSICOLOGEMAS

4 REVISTA DE SEXOLOGIA

4 SOCIOLOGIA DEL TRABAJO

4 STUDIA HISTORICA. HISTORIA ANTIGUA

4 UTOPIAS. NUESTRA BANDERA

3 ANALES DEL INSTITUTO DE ESTUDIOS MADRILEÑOS

3 ANUARIO DE PSICOLOGIA

3 ARCHIPIELAGO

3 BAETICA. ESTUDIOS DE ARTE, GEOGRAFIA E HISTORIA

3 BASILISCO, EL 
3 BOLETIN DE PSICOLOGIA

3 BOLETIN DEL INSTITUTO DE ESTUDIOS GIENNENSES

3 CUADERNOS DE GEOGRAFIA. UNIVERSIDAD DE VALENCIA

3 CUADERNOS DE PEDAGOGIA

3 ECOLOGIA POLITICA. CUADERNOS DE DEBATE INTERNACIONAL

3 GOYA

3 GRAMMA. REVISTA DE GRAFOLOGIA Y CIENCIAS AFINES

3 HISTORIA SOCIAL

3 INICIATIVA SOCIALISTA

3 INVESTIGACIONES ECONOMICAS

3 IP MARK

3 LETRAS DE DEUSTO

3 PAGINA ABIERTA

3 PAPERS. REVISTA DE SOCIOLOGIA

3 PEDRALBES. REVISTA D'HISTORIA MODERNA

3 QUIMERA

3 REVISTA DE HISTORIA DE LA PSICOLOGIA

3 REVISTA ESPAÑOLA DE DERECHO MILITAR

3 REVISTA ESPAÑOLA DE ESTUDIOS NORTEAMERICANOS

3 REVISTA INTERNACIONAL DE SOCIOLOGIA

3 TREBALLS DE LA SOCIETAT CATALANA DE GEOGRAFIA

3 TROCADERO. REVISTA DE HISTORIA MODERNA Y CONTEMPORANEA

2 ACTA HISTORICA ET ARCHAEOLOGICA MEDIAEVALIA

2 ADICCIONES. REVISTA DE SOCIDROGALCOHOL

2 AFERS. FULLS DE RECERCA I PENSAMENT

2 ANALES DE HISTORIA DEL ARTE

2 ANSIEDAD Y ESTRES

2 ANTHROPOLOGICA. REVISTA DE ETNOPSICOLOGICA Y ETNOPSIQUIATRIA

2 ANTIQUARIA

2 ANUARIO DEL DEPARTAMENTO DE HISTORIA

2 ARCHIVO ESPAÑOL DE ARTE

2 AWRAQ

2 BOLETIN DE ESTUDIOS HISTORICOS SOBRE SAN SEBASTIAN

2 BOLETIN DE LA REAL SOCIEDAD BASCONGADA DE AMIGOS DEL PAIS

2 CADERNOS DE PSICOLOXIA 
2 CONTRASTES. REVISTA DE HISTORIA MODERNA

2 CUADERNOS DE HISTORIA CONTEMPORANEA

2 CUADERNOS DE INVESTIGACION FILOLOGICA

2 CUADERNOS DE RELACIONES LABORALES

2 CUADERNOS DEL MUNDO ACTUAL

2 DESARROLLO

2 DICENDA. CUADERNOS DE FILOLOGIA HISPANICA

2 EDAD DE ORO

2 EGUZKILORE

2 EPOS. REVISTA DE FILOLOGIA

2 ES. ECONOMIA Y SOCIEDAD

2 ESPACIO, TIEMPO Y FORMA. HISTORIA MODERNA

2 ESPACIO, TIEMPO Y FORMA. SERIE VII: HISTORIA DEL ARTE

2 ESTUDIOS DE HISTORIA SOCIAL

2 ESTUDIOS DE PSICOLOGIA

2 ESTUDIOS GEOGRAFICOS

2 ESTUDIS. REVISTA DE HISTORIA MODERNA

2 FAMILIA Y SOCIEDAD

2 GADES

2 HISPANIA

2 HISPANIA ANTIQUA

2 INFAD. PSICOLOGIA DE LA INFANCIA Y LA ADOLESCENCIA

2 INTERACCION SOCIAL

2 INTERVENCION PSICOSOCTAL

2 JABEGA

2 LAPIZ. REVISTA MENSUAL DE ARTE

2 LETRA INTERNACIONAL

2 LEVIATAN. REVISTA DE HECHOS E IDEAS

2 MANUSCRITS. REVISTA D'HISTORIA MODERNA

2 POLIS

2 POLITICA EXTERIOR

2 PSI. COMUNICACION

2 PSICOLOGIA CONDUCTUAL

2 PSICOLOGIA TEXT I CONTEXT

2 RETAMA

2 REVISTA DE DERECHO PENAL Y CRIMINOLOGIA

2 REVISTA DE ESTUDIOS AGROSOCIALES 
2 REVISTA DE HISTORIA MODERNA

2 REVISTA DE PSICOTERAPIA

2 REVISTA INTERNACIONAL DEL TRABAJO

2 RTS. REVISTA DE TRABAJO SOCIAL

2 SEXPOL. REVISTA DE INFORMACION SEXOLOGICA

2 SISTEMA. REVISTA DE CIENCIAS SOCIALES

2 STUDIA ZAMORENSIA

2 TRABAJO SOCIAL HOY

2 UROGALLO, EL

2 VIENTO SUR

1 A DISTANCIA

1 ACACIA

1 ACADEMIA

1 ACTUALIDAD LABORAL

1 ADMINISTRACION DE ANDALUCIA. REVISTA ANDALUZA DE ADMINISTRACION PUBLICA

1 ALCANTARA. REVISTA DEL SEMINARIO DE ESTUDIOS CACEREÑOS

1 ALFOZ

1 ANALES DE ESTUDIOS ECONOMICOS Y EMPRESARIALES

1 ANALES DE LA FUNDACION JOAQUIN COSTA

1 ANALES DE LA LITERATURA ESPAÑOLA CONTEMPORANEA

1 ANALES DE LA REAL ACADEMIA DE JURISPRUDENCIA Y LEGISLACION

1 ANALES DE LA UNIVERSIDAD DE ALICANTE. HISTORIA MEDIEVAL

1 ANALES DE LA UNIVERSIDAD DE CADIZ

1 ANALES DE PSICOLOGIA

1 ANALES DEL MUSEO DEL PUEBLO ESPAÑOL

1 ANALES DEL SEMINARIO DE HISTORIA DE LA FILOSOFIA

1 ANALES. MUSEO DE AMERICA

1 ANALISI

1 ANTIGUEDAD Y CRISTIANISMO

1 ANUARIO DE DERECHO CIVIL

1 ANUARIO DE ESTUDIOS ATLANTICOS

1 ANUARIO DE ESTUDIOS MEDIEVALES

1 ANUARIO DE HISTORIA CONTEMPORANEA

1 ANUARIO INSTITUTO DE ESTUDIOS ZAMORANOS «FLORIAN DE OCAMPO» 
1 ARAGON EN LA EDAD MEDIA. ESTUDIOS DE ECONOMIA Y SOCIEDAD

1 ARCHIVO AGUSTINIANO. REV. DE ESTUDIOS HISTORICOS

1 ARCHIVO DE FLLOLOGIA ARAGONESA

1 ARCHIVOS DE LA FILMOTECA. REVISTA DE ESTUDIOS HISTORICOS SOBRE LA IMAGEN

1 ARCHIVOS LEONESES. REVISTA DE ESTUDIOS Y DOCUMENTACION DE LOS REINOS HISPANO-OCCIDENTALES

1 ARCHIVUM

1 ARS LONGA. CUADERNOS DE ARTE

1 ARTE, INDIVIDUO Y SOCIEDAD

1 AULA ABIERTA

1 AULA DE INNOVACION EDUCATIVA

1 AYER

1 BOLETIN AURIENSE

1 BOLETIN DE ARTE

1 BOLETIN DE LA ASOCIACION ESPAÑOLA DE ORIENTALISTAS

1 CADERNOS DO NOROESTE

1 CAMPO, EL

1 CATALINA DE ARAGON. REGINA ANGLIAE

1 CAUCE

1 CHRONICA NOVA

1 CLINICA Y ANALISIS GRUPAL

1 COGNITIVA

1 COMPLUTUM EXTRA

1 CROQUIS, EL

1 CUADERNOS DE ARTE DE LA UNIVERSIDAD DE GRANADA

1 CUADERNOS DE ARTE E ICONOGRAFIA

1 CUADERNOS DE ESTRATEGIA

1 CUADERNOS DE FILOLOGIA INGLESA

1 CUADERNOS DE HISTORIA MODERNA

1 CUADERNOS DE POLITICA CRIMINAL

1 CUADERNOS DE PSIQUIATRIA Y PSICOTERAPIA INFANTIL

1 CUADERNOS DE SECCION. HISTORIA. GEOGRAFIA

1 CUADERNOS HISPANOAMERICANOS. LOS COMPLEMENTARIOS

1 CUADERNOS REPUBLICANOS

1 CUENCA 
1 DEBATS

1 DERECHOS HUMANOS

1 DISTRIBUCION Y CONSUMO

1 DYNAMIS

1 EDUCACION Y SOCIEDAD

1 EKONOMIAZ. REVISTA DE ECONOMIA VASCA

1 EN LA ESPAÑA MEDIEVAL

1 ESIC-MARKET

1 ESPACIO, TIEMPO Y FORMA. HISTORIA CONTEMPORANEA

1 ESPACIO, TIEMPO Y FORMA. SERIE VII: HISTORIA DEL ARTE

1 ESTUDIOS DE LA ANTIGUEDAD

1 ESTUDIOS HUMANISTICOS

1 ESTUDIOS HUMANISTICOS. FILOLOGIA

1 ESTUDIOS PENALES Y CRIMINOLOGICOS

1 ESTUDIOS SOBRE EL MENSAJE PERIODISTICO

1 ESTUDIS D'HISTORIA CONTEMPORANIA DEL PAIS VALENCIA

1 FILM-HISTORIA

1 FLORENTIA ILIBERRITANA. REVISTA DE ESTUDIOS DE ANTIGÜEDAD CLASICA

1 FRANCOFONIA

1 GALLAECIA

1 HABIS

1 HISTORIA DE LA EDUCACION . REVISTA INTERUNIVERSITARIA

1 HISTORIA. INSTITUCIONES. DOCUMENTOS

1 ILERDA

1 INGURUAK. REVISTA DE SOCIOLOGIA

1 INSULA

1 INTUS. REVISTA DE LAS UNIDADES DOCENTES DE PSICOLOGIA MEDICA...

1 INVESTIGACION EN LA ESCUELA

1 INVESTIGACIONES GEOGRAFICAS

1 INVESTIGACIONES HISTORICAS. EPOCA MODERNA Y CONTEMPORANEA

1 INVESTIGACIONES PSICOLOGICAS

1 ITEM. REVISTA DE BIBLIOTECONOMIA I DOCUMENTACIO

1 IUS CANONICUM 
1 KOBIE. BELLAS ARTES

1 LIÑO

1 LLULL. BOLETIN DE LA SOCIEDAD ESPAÑOLA DE HISTORIA DE LAS CIENCIAS

1 LUCENTUM

1 MAYAB

1. METODOS DE INFORMACION

1 MILLARS. ESPAI I HISTORIA

1 MISCELANEA DE ESTUDIOS ARABES Y HEBRAICOS

1 NUMEROS

1 OBRADOIRO

1 PAPELES DE GEOGRAFIA

1 PODER JUDICIAL

1 POLIGONOS. REVISTA DE GEOGRAFIA

1 PSICOLOGIA DEL TRABAJO Y ORGANIZACIONES

1 PSICOLOGIA POLITICA

1 PUBLICACIONES. ESCUELA UNIVERSITARIA DEL PROFESORADO DE MELILLA

1 PUERTA NUEVA. REV. DE EDUCACION

1 RANDA

1 REVISTA COMPLUTENSE DE EDUCACION

1 REVISTA COMPLUTENSE DE HISTORIÁ DE AMERICA

1 REVISTA D'HISTORIA MEDIEVAL

1 REVISTA DE ESTUDIOS ANTEQUERANOS

1 REVISTA DE ESTUDIOS EXTREMEÑOS

1 REVISTA DE ESTUDIOS POLITICOS

1 REVISTA DE ESTUDIOS REGIONALES

1 REVISTA DE FILOLOGIA FRANCESA

1 REVISTA DE GIRONA

1 REVISTA DE HUMANIDADES

1 REVISTA DE INDIAS

1 REVISTA DE INSTITUCIONES EUROPEAS

1 REVISTA DE LA FACULTAD DE HUMANIDADES DE JAEN

1 REVISTA DE LITERATURA MEDIEVAL

1 REVISTA DE PSICOTERAPIA Y PSICOSOMATICA

1 REVISTA DE SERVICIOS SOCIALES Y POLITICA SOCIAL

1 REVISTA DE TRABAJO Y SEGURIDAD SOCIAL 
1 REVISTA ESPAÑOLA DE ANTROPOLOGIA AMERICANA

1 REVISTA ESPAÑOLA DE DERECHO INTERNACIONAL

1 REVISTA ESPAÑOLA DE ECONOMIA

1 REVISTA ESPAÑOLA DE TERAPIA DEL COMPORTAMIENTO

1 REVISTA GENERAL DE DERECHO

1 REVISTA IBEROAMERICANA DE EDUCACION

1 REVISTA INTERUNIVERSITARIA DE FORMACION DEL PROFESORADO

1 REVISTA JURIDICA DE ASTURIAS

1 SINTESIS. REVISTA DOCUMENTAL DE CIENCIAS SOCIALES IBEROAMERICANAS

1 SPAL. REVISTA DE PREHISTORIA Y ARQUEOLOGIA

1 STUDIUM. REVISTA DE HUMANIDADES

1 SURGAM. REVISTA DE ORIENTACION PSICOPEDAGOGICA

1 TARBIYA

1 TEMPS D'EDUCACIO

1 THEMATA

1 TIEMPO DE PAZ

1 TRABAJADORES DE LA ENSEÑANZA

1 TRABAJOS DE ARQUEOLOGIA NAVARRA

1 TRABAJOS DE PREHISTORIA

1 TRIENIO. ILUSTRACION Y LIBERALISMO. REVISTA DE HISTORIA

1 VINCULOS. REVISTA DE PSICODRAMA, TERAPIA FAMILIAR... 